hep-th/0203267

ROM2F $/ 2002 / 07$

ITEP-TH-07/02

HUTP-01/A074

\title{
IIA String Theory on Calabi-Yau Fourfolds with Background Fluxes
}

\author{
Sergei Gukov and Michael Haack \\ * Jefferson Physical Laboratory \\ Harvard University \\ Cambridge, MA 02138, USA \\ - Dipartimento di Fisica \\ Università di Roma, "Tor Vergata" \\ 00133 Rome, Italy
}

\begin{abstract}
Looking for string vacua with fixed moduli, we study compactifications of type IIA string theory on Calabi-Yau fourfolds in the presence of generic Ramond-Ramond fields. We explicitly derive the (super)potential induced by Ramond-Ramond fluxes performing a Kaluza-Klein reduction of the ten-dimensional effective action. This can be conveniently achieved in a formulation of the massive type IIA supergravity where all Ramond-Ramond fields appear in a democratic way. The result agrees with the general formula for the superpotential written in terms of calibrations. We further notice that for generic RamondRamond fluxes all geometric moduli are stabilized and one finds non-supersymmetric vacua at positive values of the scalar potential.
\end{abstract}

March 2002 


\section{Introduction and Summary}

The minimal $\mathcal{N}=1$ supersymmetry in $3+1$ dimensions is a very attractive framework for addressing many outstanding problems in theoretical high energy physics, including unification of the gauge couplings, the hierarchy problem and maybe even the cosmological constant problem. Moreover, there are phenomenological indications for minimal supersymmetry just above the electroweak scale, in the energy range of about $100 \mathrm{GeV}-1 \mathrm{TeV}$. This strongly motivates the study of string models with four supercharges.

For a long time, compactifications of the heterotic $E_{8} \times E_{8}$ string theory on CalabiYau three-folds have been the leading candidates for constructing realistic models with $\mathcal{N}=1$ supersymmetry. The other superstring theories were much less interesting; there was even a no-go theorem proving the impossibility of obtaining the Standard Model out of perturbative type II theories [1]. The situation has changed, however, with the discovery of string dualities which allow to make definite statements even in the regions of moduli space where perturbation theory cannot be used. Thus phenomenologically interesting $\mathcal{N}=1$ four-dimensional string vacua can be equivalently described as non-perturbative vacua of type IIB string theory known as F-theory compactifications on elliptically fibered Calabi-Yau fourfolds [2], or also as M-theory compactifications on singular $G_{2}$ holonomy manifolds that recently received a lot of attention.

However, all attempts to get four-dimensional $\mathcal{N}=1$ supergravity out of string or M-theory face the so called moduli problem. Typically there are a number of massless scalars in the spectrum which arise e.g. due to geometric moduli of the compactificationmanifold. A possible solution to the moduli problem lies in turning on background fluxes in the vacuum. These induce effective superpotentials of the form [3, 4]

$$
W=\int_{X} \text { Flux } \wedge \text { Calibration }
$$

with $X$ the compactification-manifold, and therefore generically lift at least part of the moduli space. Due to this phenomenological prospect type II and M-theory compactifications on Calabi-Yau threefolds with fluxes and their heterotic counterparts have attracted a lot attention in the past [5] - [30]. Also the potential induced by 4-form fluxes in compactifications of M-theory on $G_{2}$ and $\operatorname{Spin}(7)$ manifolds has been investigated [4, 31, 32, 33]. All these cases have in common that the potential can indeed be expressed via a superpotential of the form (1.1) and that it is difficult to stabilize all the moduli. 
Recently, Giddings, Kachru and Polchinski proposed an elegant way to generate large hierarchies and to stabilize some of the moduli in warped $\mathcal{N}=1$ compactifications of F-theory on Calabi-Yau fourfolds with background fluxes [25]. However, in the case of Ftheory compactifications with fluxes the Kähler moduli and specifically the volume modulus remain unfixed [25] (see however [34]). The problem is expected to be resolved by quantum corrections, which modify the classical expression for the effective potential. In string theory, there are e.g. non-perturbative corrections to the superpotential from five-brane instantons [35, 36], and it is natural to speculate that competing effects of background fluxes and five-brane instantons result in a superpotential that leads to isolated minima.

Closely related to compactifications of F-theory on Calabi-Yau fourfolds are compactifications of M-theory and type IIA string theory on the same manifolds [2]. Albeit less interesting for phenomenological applications, the resulting $\mathcal{N}=2($ resp. $\mathcal{N}=(2,2))$ supersymmetric theories in $2+1$ (resp. $1+1$ ) dimensions exhibit many similar features as $\mathcal{N}=1$ four-dimensional vacua of F-theory. Yet, they are more tractable because the low-energy physics of M-theory and IIA string theory can be described in terms of effective supergravity theories, which are suitable starting points for Kaluza-Klein reductions with background fluxes. The explicit compactification of M-theory on Calabi-Yau fourfolds with background 4-form fluxes has been studied in [37] (see also [38, 39, 3, 40, 41, 42, with the result that the potential can again be expressed via superpotentials of the form (1.1). A similar problem of direct computation of the scalar potential induced by 4 -form flux in M-theory on $G_{2}$ holonomy manifold has been addressed recently in [33].

In this paper we address the corresponding problem for type IIA compactifications on Calabi-Yau fourfolds (see also [39,43, 44, 4, 45, 46, 47]). By performing a direct KaluzaKlein reduction we explicitly derive the (super)potential induced by generic RamondRamond fluxes. As in the previous examples, we find complete agreement with the general formula (1.1). Since in type IIA theory there are more possible fluxes, the analysis is more complicated and requires a special care to treat all Ramond-Ramond fields democratically [48]. On the other hand, for the same reason, the structure of the induced superpotential is richer. In particular, it allows to stabilize all geometric moduli of the Calabi-Yau fourfold including its volume.

The main result of the paper is the explicit formula for the scalar potential (eqn. (3.23) below), which can be expressed in terms of a superpotential $W$ depending on the complex structure moduli and a twisted superpotential $\widehat{W}$ depending on the (complexified) 
Kähler moduli of the Calabi-Yau fourfold. Both of them are of the form (1.1), cf. sect. 3. In terms of $W$ and $\widehat{W}$ the potential can be expressed as:

$$
V=e^{K_{3,1}} g^{-1 \alpha \bar{\beta}} D_{\alpha} W D_{\bar{\beta}} \bar{W}+e^{K_{1,1}} g^{-1 A \bar{B}} D_{A} \widehat{W} D_{\bar{B}} \overline{\widehat{W}}
$$

where $D_{A}$ and $D_{\alpha}$ are appropriate Kähler covariant derivatives and $K_{3,1}$ (resp. $K_{1,1}$ ) is the Kähler potential of the complex structure (resp. Kähler moduli). Note, that the potential splits into one part for the complex structure moduli and one for the Kähler moduli. Furthermore, similar to the four-dimensional [25] and three-dimensional [37] case, eqn. (1.2) does not contain any $|W|^{2}$ and $|\widehat{W}|^{2}$ terms.

It would be interesting to embed our analysis in a manifestly supersymmetric description of the effective two-dimensional theory. A natural framework for this would be a superspace description of the effective $\mathcal{N}=(2,2)$ supergravity coupled to matter, e.g. as in the hybrid description of the superstring [47] or in the new two-dimensional dilaton supergravity [45]. Even though such a description can be obtained in the case without potential, we were not able to incorporate the superpotentials in $\mathcal{N}=(2,2)$ superspace. Nevertheless, in the appendix we derive the chiral density projectors in the ungauged $\mathcal{N}=(2,2)$ dilaton supergravity of [45], which might not only play a role in solving this problem, but also be of independent interest in the general study of two-dimensional supergravity in superspace [45, 49, 50,51].

The organization of the paper is as follows. In section 2, using the dual formulation of [48], we derive a form of the ten-dimensional massive type IIA theory, which breaks the ten-dimensional covariance but serves as a convenient starting point for a compactification on a Calabi-Yau fourfold with fluxes. This compactification is done in section 3, where the potential due to the Ramond-Ramond background fluxes is derived. The derivation also takes into account some of the higher derivative corrections to the type IIA action. Furthermore we find a more general tadpole cancellation condition than in three dimensions. At the end of this section we make some comments about the vacuum structure of the two-dimensional theory. Like in compactifications to three dimensions, we find no stable AdS or dS vacua. On the other hand, there are more possibilities to break supersymmetry and stabilize moduli than in $2+1$ and $3+1$ dimensions. In section 4 we illustrate the rather technical derivation in section 3 by looking at three cases, in which only a single type of RR-field strength has a background value. Specifically, we consider the cases of non-vanishing 0-, 8- and 4-form flux. This should help the reader to understand the 
physical implications of the different fluxes and the connection to the three-dimensional case, in which only 4-form flux can appear in the background. Finally, we have included two appendices. The first one collects some of our conventions and useful formulas. In appendix II (which can be read independently of the rest of the paper) we derive the chiral and twisted chiral density projectors in $\mathcal{N}=(2,2)$ two-dimensional dilaton supergravity.

\section{A Dual Formulation of Massive Type IIA Theory}

In this section we work out a formulation of Type IIA supergravity suitable for compactifications on 8-manifolds, which we will use to perform a compactification on a CalabiYau fourfold $Y_{4}$ in the next section. The effective action we are going to obtain breaks the full ten-dimensional covariance, and makes a $2+8$ split of the coordinates. However, it has the same degrees of freedom as the usual (massive) Type IIA theory, albeit written in a different, dual language.

The standard bosonic action of the massive Type IIA supergravity looks like [52]:

$$
\begin{aligned}
S_{10} & =\frac{1}{2 \kappa_{10}^{2}} \int d^{10} x \sqrt{-g}\left[e^{-2 \varphi}\left(R+4|\partial \varphi|^{2}-\frac{1}{2}|H|^{2}\right)-\frac{1}{2} \sum_{n=0,1,2}\left|G_{2 n}\right|^{2}\right]- \\
& -\frac{1}{4 \kappa_{10}^{2}} \int\left(d C_{3} d C_{3} B+\frac{1}{3} G_{0} d C_{3} B^{3}+\frac{1}{20} G_{0}^{2} B^{5}\right)+\ldots,
\end{aligned}
$$

where we omit the wedge products and use the abbreviations

$$
\begin{aligned}
G_{0} & =\text { mass parameter } \\
G_{2} & =d C_{1}+G_{0} B \\
G_{4} & =d C_{3}-C_{1} d B+\frac{1}{2} G_{0} B^{2} .
\end{aligned}
$$

Furthermore for a $p$-form $\omega_{p}$ we use the notation $|\omega|^{2}=\frac{1}{p !} \omega_{M_{1} \ldots M_{p}} \omega^{M_{1} \ldots M_{p}}$ and the dots in (2.1) stand for higher derivative terms. This standard action can be obtained via integrating out the fields $A_{(2 n-1)}$ in the dual action [48]:

$$
\begin{aligned}
S_{10} & =\frac{1}{2 \kappa_{10}^{2}} \int d^{10} x \sqrt{-g}\left[e^{-2 \varphi}\left(R+4|\partial \varphi|^{2}-\frac{1}{2}|H|^{2}\right)-\frac{1}{2} \sum_{n=0,1,2}\left|G_{2 n}\right|^{2}\right]- \\
& -\frac{1}{4 \kappa_{10}^{2}} \int\left(G_{4} G_{4} B-G_{2} G_{4} B^{2}+\frac{1}{3} G_{2}^{2} B^{3}+\frac{1}{3} G_{0} G_{4} B^{3}-\frac{1}{4} G_{0} G_{2} B^{4}+\right. \\
& \left.+\frac{1}{20} G_{0}^{2} B^{5}+2 e^{-B} \mathbf{G} d\left(A_{5}-A_{7}+A_{9}\right)\right)+\ldots,
\end{aligned}
$$


where

$$
\mathbf{G}=G_{0}+G_{2}+G_{4}
$$

is a formal sum of Ramond-Ramond fields, which are however a priori independent fields, not given by (2.2) (compare the discussion in [48]). Moreover, in the last term of (2.3) a projection on the 10-form part is understood. The equations of motion for $A$ now impose the following Bianchi identities for $\mathbf{G}$ :

$$
d\left(e^{-B} \wedge \mathbf{G}\right)=0
$$

This set of equations has the general solution 1

$$
e^{-B} \wedge \mathbf{G}=d \mathbf{A}
$$

This can be expressed in components:

$$
\begin{aligned}
& G_{0}=\text { mass parameter } \\
& G_{2}=d A_{1}+G_{0} B \\
& G_{4}=d A_{3}+G_{2} B-\frac{1}{2} G_{0} B^{2} .
\end{aligned}
$$

Converting these into the "C-basis"

$$
\mathbf{A}=\mathbf{C} \wedge e^{-B}
$$

and substituting back into (2.3), we get the standard Type IIA action (2.1).

In principle it would also be possible to integrate out the fields $\mathbf{G}$ in (2.3) keeping $A_{5}, A_{7}$ and $A_{9}$. However, in view of our aim to perform a Calabi-Yau fourfold compactification with background fluxes, we want to proceed in a different way, keeping some components of the $A$ s and some of the Gs. To be more specific, we distinguish two of the coordinates $\left(x^{\mu}, \mu=1,2\right)$ from the others $\left(x^{a}, a=1, \ldots, 8\right)$, denoting them as 'external' since they will be the uncompactified coordinates in the $Y_{4}$-compactification in the next section. At this stage this name helps us to distinguish them from the $x^{a}$. What we have in mind now is to integrate out all components from $\mathbf{G}$ in (2.3) with two external indices,

$1 \mathbf{A}=A_{1}+A_{3}$ is a formal sum like $\mathbf{G}$ in (2.4). Furthermore the $A$ s are not necessarily globally defined. In the presence of background fluxes considered in the next section, $d \mathbf{A}$ is a nontrivial cohomology element. 
keeping those with zero or one (and instead integrating out the corresponding components of $A_{5}, A_{7}$ and $A_{9}$ ). This leads to an action which is (classically) equivalent to (2.1) and has the same degrees of freedom but expressed in different variables, which are more adapted to a Calabi-Yau fourfold compactification with background fluxes.

In order to carry out this program we have to introduce some notation. For any $p$-form we define

$$
\begin{aligned}
& \omega_{p}^{(0)}=\frac{1}{p !} \omega_{a_{1} \ldots a_{p}} d x^{a_{1}} \wedge \ldots \wedge d x^{a_{p}} \\
& \omega_{p}^{(1)}=\frac{1}{(p-1) !} \omega_{\mu a_{1} \ldots a_{p-1}} d x^{\mu} \wedge d x^{a_{1}} \wedge \ldots \wedge d x^{a_{p-1}} \\
& \omega_{p}^{(2)}=\frac{1}{(p-2) !} \frac{1}{2 !} \omega_{\mu \nu a_{1} \ldots a_{p-2}} d x^{\mu} \wedge d x^{\nu} \wedge d x^{a_{1}} \wedge \ldots \wedge d x^{a_{p-2}}
\end{aligned}
$$

so that

$$
\omega_{p}=\omega_{p}^{(0)}+\omega_{p}^{(1)}+\omega_{p}^{(2)}
$$

Furthermore, we define exterior derivatives

$$
\begin{aligned}
& d^{(0)} \omega_{p}^{(0)}=\frac{1}{p !} \frac{\partial}{\partial x^{b}} \omega_{a_{1} \ldots a_{p}} d x^{b} \wedge d x^{a_{1}} \wedge \ldots \wedge d x^{a_{p}} \\
& d^{(1)} \omega_{p}^{(0)}=\frac{1}{p !} \frac{\partial}{\partial x^{\mu}} \omega_{a_{1} \ldots a_{p}} d x^{\mu} \wedge d x^{a_{1}} \wedge \ldots \wedge d x^{a_{p}}
\end{aligned}
$$

and similarly for $\omega_{p}^{(1)}$ and $\omega_{p}^{(2)}$, such that $d=d^{(0)}+d^{(1)}$.

Now we decompose all forms $G_{2}, G_{4}, A_{5}, A_{7}, A_{9}$ and $B$ as in (2.10). Before substituting these decompositions into the action (2.3), it is convenient to write the Lagrangian as a sum

$$
L_{10}=T_{N S-N S}+T_{R-R}+L_{C S}
$$

where

$$
\begin{aligned}
T_{N S-N S} & =d^{10} x \sqrt{-g} e^{-2 \varphi}\left(R+4|\partial \varphi|^{2}-\frac{1}{2}|H|^{2}\right), \\
T_{R-R} & =-\frac{1}{2} d^{10} x \sqrt{-g} \sum_{n=0,1,2}\left|G_{2 n}\right|^{2}=-\frac{1}{2} \sum_{n=0,1,2} G_{2 n} \wedge \star G_{2 n},
\end{aligned}
$$

and $L_{C S}$ are the Chern-Simons terms in (2.3), such that $S_{10}=\frac{1}{2 \kappa_{10}^{2}} \int L_{10} \cdot T_{N S-N S}$ are the kinetic terms for the NSNS-fields and $T_{R-R}$ will become those for the RR-fields after integrating out the corresponding $A$-fields. 
Plugging the decomposition of the $G$ s and $A$ s into (2.12) results in the following expressions

$$
T_{R-R}=-\frac{1}{2} G_{0} \wedge \star G_{0}-\frac{1}{2} \sum_{n=1,2} \sum_{k=0,1,2} G_{2 n}^{(k)} \wedge \star G_{2 n}^{(k)}
$$

and

$$
\begin{aligned}
L_{C S}= & G_{2}^{(2)}\left(\frac{1}{2} G_{4}^{(0)}\left(B^{(0)}\right)^{2}-\frac{1}{3} G_{2}^{(0)}\left(B^{(0)}\right)^{3}+\frac{1}{8} G_{0}\left(B^{(0)}\right)^{4}+d^{(0)} A_{7}^{(0)}+B^{(0)} d^{(0)} A_{5}^{(0)}\right) \\
& +G_{4}^{(2)}\left(-G_{4}^{(0)} B^{(0)}+\frac{1}{2} G_{2}^{(0)}\left(B^{(0)}\right)^{2}-\frac{1}{6} G_{0}\left(B^{(0)}\right)^{3}-d^{(0)} A_{5}^{(0)}\right) \\
& -d^{(0)} A_{9}^{(2)} G_{0}-d^{(1)} A_{9}^{(1)} G_{0} \\
& +\left(d^{(0)} A_{7}^{(2)}+d^{(1)} A_{7}^{(1)}\right)\left(G_{2}^{(0)}-G_{0} B^{(0)}\right)+d^{(0)} A_{7}^{(1)}\left(G_{2}^{(1)}-G_{0} B^{(1)}\right) \\
& +\left(d^{(0)} A_{5}^{(2)}+d^{(1)} A_{5}^{(1)}\right)\left(-G_{4}^{(0)}+G_{2}^{(0)} B^{(0)}-\frac{1}{2} G_{0}\left(B^{(0)}\right)^{2}\right) \\
& +d^{(0)} A_{5}^{(1)}\left(-G_{4}^{(1)}+G_{2}^{(1)} B^{(0)}+G_{2}^{(0)} B^{(1)}-G_{0} B^{(0)} B^{(1)}\right) \\
& +d^{(1)} A_{7}^{(0)} G_{2}^{(1)}-d^{(1)} A_{5}^{(0)}\left(G_{4}^{(1)}-G_{2}^{(1)} B^{(0)}\right) \\
& +B^{(2)} X_{(1)}+\left(B^{(1)}\right)^{2} X_{(2)}+B^{(1)} X_{(3)}+B^{(0)} X_{(4)},
\end{aligned}
$$

where

$$
\begin{aligned}
X_{(1)}= & -\frac{1}{2}\left(G_{4}^{(0)}-G_{2}^{(0)} B^{(0)}+\frac{1}{2} G_{0}\left(B^{(0)}\right)^{2}\right)^{2}+\left(G_{2}^{(0)}-G_{0} B^{(0)}\right) d^{(0)} A_{5}^{(0)}-G_{0} d^{(0)} A_{7}^{(0)}, \\
X_{(2)}= & \frac{1}{2} G_{2}^{(0)} G_{4}^{(0)}-\frac{1}{2}\left(G_{2}^{(0)}\right)^{2} B^{(0)}-\frac{1}{2} G_{0} G_{4}^{(0)} B^{(0)}+\frac{3}{2} G_{0} G_{2}^{(0)}\left(B^{(0)}\right)^{2} \\
& -\frac{1}{2} G_{0}^{2}\left(B^{(0)}\right)^{3}-\frac{1}{2} G_{0} d^{(0)} A_{5}^{(0)} \\
X_{(3)}= & -\left(G_{4}^{(0)}-G_{2}^{(0)} B^{(0)}+\frac{1}{2} G_{0}\left(B^{(0)}\right)^{2}\right)\left(G_{4}^{(1)}-G_{2}^{(1)} B^{(0)}\right)-G_{0} d^{(1)} A_{7}^{(0)} \\
& +\left(G_{2}^{(0)}-G_{0} B^{(0)}\right) d^{(1)} A_{5}^{(0)}+G_{2}^{(1)} d^{(0)} A_{5}^{(0)} \\
X_{(4)}= & -\frac{1}{2} G_{4}^{(1)}\left(G_{4}^{(1)}-G_{2}^{(1)} B^{(0)}\right)-\frac{1}{6}\left(G_{2}^{(1)}\right)^{2}\left(B^{(0)}\right)^{2},
\end{aligned}
$$

which only contain $A^{(0)}$ s.

Integrating out $A_{5}^{(2)}, A_{7}^{(2)}$ and $A_{9}^{(2)}$ (occurring in the third to fifth line of (2.15)), i.e. those components with two external indices, leads to a similar solution as in (2.7). To be more precise we get

$$
\begin{aligned}
G_{0} & =\text { independent of } x^{a} \\
G_{2}^{(0)} & =d^{(0)} A_{1}^{(0)}+G_{0} B^{(0)} \\
G_{4}^{(0)} & =d^{(0)} A_{3}^{(0)}+G_{2}^{(0)} B^{(0)}-\frac{1}{2} G_{0}\left(B^{(0)}\right)^{2} .
\end{aligned}
$$


With the help of (2.17) the expressions $X_{(1)}, X_{(2)}$ and $X_{(3)}$ from (2.16) simplify and become

$$
\begin{aligned}
& X_{(1)}=-\frac{1}{2}\left(d^{(0)} A_{3}^{(0)}\right)^{2}+d^{(0)} A_{1}^{(0)} d^{(0)} A_{5}^{(0)}-G_{0} d^{(0)} A_{7}^{(0)}, \\
& X_{(2)}=-\frac{1}{2} G_{0} d^{(0)} A_{5}^{(0)}+\frac{1}{2} d^{(0)} A_{1}^{(0)} d^{(0)} A_{3}^{(0)}+\frac{3}{4} G_{0} d^{(0)} A_{1}^{(0)}\left(B^{(0)}\right)^{2}+\frac{1}{2} G_{0}^{2}\left(B^{(0)}\right)^{2}, \\
& X_{(3)}=-d^{(0)} A_{3}^{(0)}\left(G_{4}^{(1)}-G_{2}^{(1)} B^{(0)}\right)+d^{(0)} A_{1}^{(0)} d^{(1)} A_{5}^{(0)}-G_{0} d^{(1)} A_{7}^{(0)}+G_{2}^{(1)} d^{(0)} A_{5}^{(0)} .
\end{aligned}
$$

Furthermore integrating out $A_{5}^{(1)}, A_{7}^{(1)}$ and $A_{9}^{(1)}$ (occurring in the third to sixth line of (2.15)), i.e. those components with one external index, leads to the following constraints:

$$
\begin{aligned}
& G_{0}=\text { independent of } x^{\mu}, \\
& d^{(0)}\left(G_{2}^{(1)}-G_{0} B^{(1)}+d^{(1)} A_{1}^{(0)}\right)=0, \\
& d^{(0)}\left(G_{4}^{(1)}-G_{2}^{(1)} B^{(0)}-d^{(0)} A_{1}^{(0)} B^{(1)}+d^{(1)} A_{3}^{(0)}\right)=0,
\end{aligned}
$$

where the first equation implies, that the mass parameter is (locally) independent of the two external coordinates and in the last two equations (2.17) has been used. They are locally solved by

$$
\begin{aligned}
& G_{2}^{(1)}=d^{(0)} A_{1}^{(1)}-d^{(1)} A_{1}^{(0)}+G_{0} B^{(1)} \\
& G_{4}^{(1)}=d^{(0)} A_{3}^{(1)}-d^{(1)} A_{3}^{(0)}+G_{2}^{(1)} B^{(0)}+d^{(0)} A_{1}^{(0)} B^{(1)} .
\end{aligned}
$$

The next step is to integrate out components of the fields $\mathbf{G}$ with two external indices, i.e. $G_{2}^{(2)}$ and $G_{4}^{(2)}$. The corresponding variations of the action $S_{10}$ lead to the following equations of motion (c.f. the first two lines of (2.15)):

$$
* G_{2}^{(2)}=\frac{1}{2} G_{4}^{(0)}\left(B^{(0)}\right)^{2}-\frac{1}{3} G_{2}^{(0)}\left(B^{(0)}\right)^{3}+\frac{1}{8} G_{0}\left(B^{(0)}\right)^{4}+d^{(0)} A_{7}^{(0)}+B^{(0)} d^{(0)} A_{5}^{(0)}
$$

and

$$
* G_{4}^{(2)}=-G_{4}^{(0)} B^{(0)}+\frac{1}{2} G_{2}^{(0)}\left(B^{(0)}\right)^{2}-\frac{1}{6} G_{0}\left(B^{(0)}\right)^{3}-d^{(0)} A_{5}^{(0)} .
$$

Thus we are left with $G_{0}, A_{1}^{(0)}, A_{1}^{(1)}, A_{3}^{(0)}, A_{3}^{(1)}, A_{5}^{(0)}$ and $A_{7}^{(0)}$ as the independent fields in the RR-sector. They comprise a complete basis of independent RR-fields in the tendimensional space time. All other components, e.g. those with two external legs, can be expressed as duals of them.

Introducing the notation

$$
G_{6}^{(0)}=-* G_{4}^{(2)}, \quad G_{8}^{(0)}=* G_{2}^{(2)}
$$


it is easily verified that the relations (2.17), (2.21), (2.22) between $A_{2 n-1}^{(0)}$ and $G_{2 n}^{(0)}$ take the compact form

$$
d^{(0)} \mathbf{A}^{(0)}=e^{-B^{(0)}} \wedge \mathbf{G}^{(0)}
$$

which is similar to (2.6), but now involves only forms with no external components and also contains forms of degree six and eight:

$$
\mathbf{G}^{(0)}=\sum_{n=0}^{4} G_{2 n}^{(0)}, \quad \mathbf{A}^{(0)}=\sum_{n=1}^{4} A_{2 n-1}^{(0)} .
$$

In $D$-dimensional Minkowski space a $p$-form transforms under Hodge duality according to

$$
*\left(* \omega_{p}\right)=(-1)^{p(D-p)+1} \omega_{p} .
$$

Therefore, we can rewrite 2.23 as

$$
G_{4}^{(2)}=* G_{6}^{(0)}, \quad G_{2}^{(2)}=-* G_{8}^{(0)} .
$$

Now we substitute (2.23) and (2.27) into the Lagrangian (2.12). From $T_{R-R}$ we get:

$$
T_{R-R}=-\frac{1}{2} G_{2}^{(2)} \wedge * G_{2}^{(2)}-\frac{1}{2} G_{4}^{(2)} \wedge * G_{4}^{(2)}=\frac{1}{2} G_{8}^{(0)} \wedge * G_{8}^{(0)}+\frac{1}{2} G_{6}^{(0)} \wedge * G_{6}^{(0)}
$$

There is a further contribution to the kinetic terms of $G_{6}^{(0)}$ and $G_{8}^{(0)}$, coming from the Chern-Simons terms:

$$
L_{C S}=G_{2}^{(2)} \wedge * G_{2}^{(2)}+G_{4}^{(2)} \wedge * G_{4}^{(2)}+\ldots=-G_{8}^{(0)} \wedge * G_{8}^{(0)}-G_{6}^{(0)} \wedge * G_{6}^{(0)}+\ldots
$$

where we have used (2.21) and (2.22) in the first two lines of (2.15). Note, that the sum of the two contributions (2.28) and (2.29) leads to the right sign for the kinetic terms of $G_{6}^{(0)}$ and $G_{8}^{(0)}$.

Altogether we end up with the following expression for the dual action:2

$$
\begin{aligned}
S_{10}= & \frac{1}{2 \kappa_{10}^{2}} \int d^{10} x \sqrt{-g}\left(e^{-2 \varphi}\left(R+4|\partial \varphi|^{2}-\frac{1}{2}|H|^{2}\right)-\frac{1}{2} \sum_{n=0}^{4}\left|G_{2 n}^{(0)}\right|^{2}-\frac{1}{2} \sum_{n=1}^{2}\left|G_{2 n}^{(1)}\right|^{2}\right) \\
& +\frac{1}{2 \kappa_{10}^{2}} \int\left(B^{(2)} X_{(1)}+\left(B^{(1)}\right)^{2} X_{(2)}+B^{(1)} X_{(3)}+B^{(0)} X_{(4)}\right. \\
& \left.+d^{(1)} A_{7}^{(0)} G_{2}^{(1)}-d^{(1)} A_{5}^{(0)}\left(G_{4}^{(1)}-G_{2}^{(1)} B^{(0)}\right)\right)+\ldots
\end{aligned}
$$

2 We have only considered the bosonic part of the action here, which is all we need for a derivation of the potential. We assume that the procedure can be made supersymmetric. 
where $X_{(1)}, \ldots, X_{(4)}$ are given in (2.16) respectively $(2.18), G_{2 n}^{(1)}$ are defined in (2.20) and $G_{2 n}^{(0)}$ are given in (2.24), which can be inverted to 3

$$
\mathbf{G}^{(0)}=d^{(0)} \mathbf{A}^{(0)} \wedge e^{B^{(0)}}
$$

\section{3. (Super)Potential Induced By Background Ramond-Ramond Fields}

In this section we use the Type IIA action (2.30) to obtain the two-dimensional effective potential induced by background RR-fields in a Kaluza-Klein reduction on a CalabiYau fourfold. Following a similar analysis in M-theory [37], we show that the result can be expressed in terms of the superpotential for chiral moduli superfields proposed in [3],

$$
W\left(Z^{\alpha}\right)=\frac{1}{2 \pi} \int_{Y_{4}} F_{4} \wedge \Omega,
$$

where $\Omega$ is the unique $(4,0)$-form of $Y_{4}$, and for twisted chiral moduli superfields [4]:

$$
\begin{aligned}
\widetilde{W}\left(t^{A}\right) & =\frac{1}{2 \pi} \int_{Y_{4}} e^{-i \mathcal{K}} \wedge \mathcal{F}= \\
& =\frac{1}{2 \pi} \int_{Y_{4}}\left(\frac{1}{4 !} F_{0} \mathcal{K}^{4}+i \frac{1}{3 !} F_{2} \wedge \mathcal{K}^{3}-\frac{1}{2 !} F_{4} \wedge \mathcal{K}^{2}-i F_{6} \wedge \mathcal{K}+F_{8}\right)
\end{aligned}
$$

where $\mathcal{K}$ is a complexified Kähler form on $Y_{4}$.

Before we derive the potential, however, let us first recall the compactification on a Calabi-Yau fourfold $Y_{4}$ without background fluxes [46] and see how it is reproduced in the dual formulation.

\subsection{Reduction Without Fluxes}

Starting point for the reduction is the action (2.30), albeit with $G_{0}=0$. We take the space-time to be of the form $\mathbb{R}^{1,1} \times Y_{4}$, where $x^{\mu}$ are the coordinates of $\mathbb{R}^{1,1}$ and $x^{a}$ are those on the Calabi-Yau fourfold $Y_{4}$. 4 The spectrum of the $D=2$ theory is determined by the deformations of the Calabi-Yau metric and the expansion of $B, A_{1}^{(0)}, A_{1}^{(1)}, A_{3}^{(0)}, A_{3}^{(1)}, A_{5}^{(0)}$

3 In order to get the correct $G_{0}$-dependence in this formula, one formally has to introduce $A_{-1}^{(0)}$ with a 0-form "field strength" $d^{(0)} A_{-1}^{(0)}$.

4 However, the determination of the massless spectrum and effective action in $D=2$ makes use of the fact, that Calabi-Yau manifolds are complex manifolds. We therefore introduce complex coordinates $\xi^{i}$ on $Y_{4}$. 
and $A_{7}^{(0)}$ in terms of the non-trivial forms of $Y_{4}$. The deformations of the metric comprise $h^{1,1}$ real Kähler deformations $M^{A}, A=1, \ldots, h^{1,1}$, and $h^{1,3}$ complex deformations $Z^{\alpha}, \alpha=$ $1, \ldots, h^{1,3}$, of the complex structure. Since vectors contain no physical degree of freedom in $D=2$ the modes $B^{(1)}, A_{1}^{(1)}$ and $A_{3}^{(1)}$ are non-dynamical. Furthermore since there are no 1- and 7-forms on $Y_{4}$ also $A_{1}^{(0)}$ and $A_{7}^{(0)}$ do not contribute any massless mode in $D=2$. $B^{(0)}$ leads to $h^{1,1}$ real scalar fields $a^{A}$, whereas expanding $A_{3}^{(0)}$ into the 3 -forms and $A_{5}^{(0)}$ into the 5 -forms of $Y_{4}$, one immediately verifies, that all $G_{2 n}^{(0)}$ vanish, as do $G_{2}^{(1)}, X_{(1)}, X_{(2)}$ and $X_{(3)}$. The only contribution from the RR-sector comes from

$$
S_{10}^{(R R)}=-\frac{1}{4 \kappa_{10}^{2}} \int d^{10} x \sqrt{-g}\left|G_{4}^{(1)}\right|^{2}-\frac{1}{2 \kappa_{10}^{2}} \int\left(\frac{1}{2} B^{(0)}\left(G_{4}^{(1)}\right)^{2}+d^{(1)} A_{5}^{(0)} G_{4}^{(1)}\right),
$$

where

$$
G_{4}^{(1)}=-d^{(1)} A_{3}^{(0)}
$$

see (2.20). In this case $A_{5}^{(0)}$ is just an auxiliary field, because it does not have a kinetic term. However, in view of (3.4), its equation of motion $d^{(1)} G_{4}^{(1)}=0$ is trivially fulfilled. Thus the decomposition of $A_{3}^{(0)}$ into the (1,2)-forms of $Y_{4}$ leads to $h^{1,2}$ complex scalars $N^{I}, I=1, \ldots, h^{1,2}$, with the same kinetic and interaction terms as in the reduction of the usual action [46]. The (1,1)-moduli reside in twisted chiral multiplets, while all other scalars are members of chiral multiplets. In the presence of both, chiral and twisted chiral multiplets, the moduli space is in general not Kähler anymore [53].

For simplicity let us discuss here only the case where the $(2,1)$-moduli are frozen to some fixed value and refer to [46] for a discussion of the general case. Compactification of the NS-part of the action (2.30) results in

$S_{2}=\frac{1}{2 \kappa_{10}^{2}} \int d^{2} x \sqrt{-g} e^{-2 \varphi^{(2)}}\left(R^{(2)}+4 \partial_{\mu} \varphi^{(2)} \partial^{\mu} \varphi^{(2)}-2 g_{A \bar{B}} \partial_{\mu} t^{A} \partial^{\mu} \bar{t}^{B}-2 g_{\alpha \bar{\beta}} \partial_{\mu} Z^{\alpha} \partial^{\mu} \bar{Z}^{\bar{\beta}}\right)$,

where the following definitions have been used [0 [45, 54]:

$$
\begin{aligned}
e^{-2 \varphi^{(2)}} & =e^{-2 \varphi} \mathcal{V}, \\
t^{A} & =M^{A}+i a^{A}, \\
g_{A \bar{B}} & =\frac{1}{4 \mathcal{V}} \int_{Y_{4}} e_{A} \wedge * e_{B}=-\partial_{A} \bar{\partial}_{\bar{B}} \ln \mathcal{V}, \\
g_{\alpha \bar{\beta}} & =-\frac{\int_{Y_{4}} \Phi_{\alpha} \wedge \bar{\Phi}_{\bar{\beta}}}{\int_{Y_{4}} \Omega \wedge \bar{\Omega}}=-\partial_{\alpha} \bar{\partial}_{\bar{\beta}} \ln \left(\int_{Y_{4}} \Omega \wedge \bar{\Omega}\right) .
\end{aligned}
$$

5 Notice the different normalization for the Kähler moduli as compared to [46]. This results in an additional factor of $\frac{1}{2}$ in the definition of $g_{A \bar{B}}$ as compared to [37, 46, 54]. 
Here $e_{A}$ denotes a basis for the $(1,1)$-forms of $Y_{4}$, whereas $\Phi_{\alpha}$ a basis for its $(3,1)$-forms. $\mathcal{V}$ is the volume of the fourfold, which can be expressed in terms of the Kähler form $J=M^{A} e_{A}$ as

$$
\mathcal{V}=\frac{1}{4 !} \int_{Y_{4}} J \wedge J \wedge J \wedge J=\frac{1}{2^{4} 4 !} d_{A B C D}\left(t^{A}+\bar{t}^{A}\right)\left(t^{B}+\bar{t}^{B}\right)\left(t^{C}+\bar{t}^{C}\right)\left(t^{D}+\bar{t}^{D}\right)
$$

where $d_{A B C D}$ are the classical intersection numbers

$$
d_{A B C D}=\int_{Y_{4}} e_{A} \wedge e_{B} \wedge e_{C} \wedge e_{D}
$$

The moduli space factorizes into chiral and twisted chiral multiplets and is Kähler despite the presence of both kinds of multiplets. The Kähler potential can be read off from (3.6) and is given by

$$
K=K_{3,1}+K_{1,1}=-\ln \left(\int_{Y_{4}} \Omega \wedge \bar{\Omega}\right)-\ln \mathcal{V}
$$

Using this Kähler potential, the total kinetic action (3.5) can be written in a manifestly supersymmetric form [45]:

$$
S=\int d^{2} x \int d^{2} \theta d^{2} \bar{\theta} E^{-1} e^{-2 V} e^{-K},
$$

where $V$ is a real superfield in $\mathcal{N}=(2,2)$ dilaton supergravity. Its leading component can be identified with the two-dimensional dilaton field.

This completes our brief review of the kinetic action for the moduli fields in the effective $\mathcal{N}=(2,2)$ two-dimensional supergravity. We refer the reader to 45, 46, 54 for further details and a discussion of related aspects.

\subsection{Reduction With Fluxes}

We now come to the main issue of this paper, to the derivation of the effective potential induced by RR-background fluxes in a Calabi-Yau fourfold compactification of massive type IIA string theory. The conditions for unbroken supersymmetry in this case have been analyzed in [⿴囗十, where also a background flux for the NS 3-form field strength has been taken into account. In order to preserve maximal symmetry of the two-dimensional space-time it has to take the form $H_{\mu \nu m}=\epsilon_{\mu \nu} \partial_{m} f$, where $f$ is a locally defined function of the internal coordinates. Furthermore the space-time metric takes the form of a warped 
product between a two-dimensional maximally symmetric space and the internal CalabiYau manifold. It is shown in [4] that the function $f$ is actually related via supersymmetry to the warp factor $\Delta$ and the two-dimensional dilaton (see eq. (I.15) of that paper). However, we do not consider possible effects of the warp-factor on the potential here and in addition consider the dilaton to be independent of the internal coordinates. Thus we concentrate on the contributions from the kinetic terms of the RR-fields, the Chern-Simons terms and some higher derivative terms, assuming an unwarped metric in the derivation.6

There are two higher derivative terms relevant for the derivation of the potential. The first correction to (2.1) is given by [55,56]

$$
\delta S_{1}=-T_{1} \int B \wedge X_{8}
$$

where $T_{1}=\left(2 \pi \alpha^{\prime}\right)^{-1}$ is the string tension and

$$
X_{8}=\frac{1}{192(2 \pi)^{4}}\left[\operatorname{tr} R^{4}-\frac{1}{4}\left(\operatorname{tr} R^{2}\right)^{2}\right], \quad \int_{Y_{4}} X_{8}=-\frac{\chi}{24} .
$$

In a Calabi-Yau fourfold compactification $\delta S_{1}$ leads to a tadpole for $B^{(2)}$ if the fourfold has non-vanishing Euler number [57,58]. This tadpole can be cancelled either by introducing space-time filling strings into the vacuum, a possibility that we do not pursue here, or by turning on RR-background fluxes, as can be seen from the term $\sim B^{(2)} X_{(1)}$ in (2.30) taken into account (2.18). Thus if we denote the background fluxes by

$$
F_{2 n}=d^{(0)} A_{2 n-1}^{(0)}
$$

the tadpole cancellation condition in the absence of space-time filling strings is

$$
\frac{1}{4 \kappa_{10}^{2}} \int_{Y_{4}}\left(2 F_{0} \wedge F_{8}-2 F_{2} \wedge F_{6}+F_{4} \wedge F_{4}\right)=\frac{T_{1}}{24} \chi
$$

which is a generalization of the three-dimensional tadpole condition involving only the 4-form background [57,58,38. Since

$$
2 \kappa_{10}^{2}=(2 \pi)^{7} \alpha^{4}
$$

6 The warp-factor actually becomes constant in the large volume limit 25,41. However, in contrast to the three-dimensional case [37], it is difficult to argue that the contributions of the warp-factor are subleading in $\alpha^{\prime}$, as we will see momentarily. It would be nice to get a better understanding of the role of the warp-factor.

7 To get a uniform notation we also apply this definition to the 0 -form background, i.e. $F_{0} \equiv$ $G_{0}$, compare the footnote at the end of section 2 . 
we see from (3.14), that the fluxes are of the order $F_{2 n} \sim \mathcal{O}\left(\alpha^{\prime n-1 / 2}\right)$, which is in agreement with the usual flux quantization conditions.8

The second correction to (2.1), that contributes to the effective potential in $D=2$, is [59,60, 61, 62, 63, 64, 65, 66, 67,68]

$$
\delta S_{2}=-\frac{b_{1}}{4 \pi \alpha^{\prime}} \int d^{10} x \sqrt{-g^{(10)}} E_{8}
$$

where $b_{1}^{-1} \equiv(2 \pi)^{4} 3^{2} 2^{13}$ (we use the conventions of [69]) and

$$
E_{8}=\frac{1}{2} \epsilon^{A B M_{1} N_{1} \ldots M_{4} N_{4}} \epsilon_{A B M_{1}^{\prime} N_{1}^{\prime} \ldots M_{4}^{\prime} N_{4}^{\prime}} R^{M_{1}^{\prime} N_{1}^{\prime}} M_{1} N_{1} \ldots R^{M_{4}^{\prime} N_{4}^{\prime}} M_{4} N_{4} .
$$

$E_{8}$ is a 10-dimensional generalization of the Euler density. Thus, in a fourfold compactification this term contributes to the potential according to [69]

$$
-\frac{b_{1}}{4 \pi \alpha^{\prime}} \int d^{10} x \sqrt{-g^{(10)}} E_{8}=\frac{T_{1}}{24} \int d^{2} x \sqrt{-g^{(2)}} \chi+\ldots
$$

where the dots stand for a correction to the two-dimensional Einstein-Hilbert term, that can be derived along the same lines as in [37] and which leads to a renormalization of the two-dimensional dilaton. However, this is not important for our discussion of the potential. Furthermore, the coefficient of the $E_{8}$-term in (3.16) does not get any higher string loop corrections [70], such that the coefficient of the Euler term in the effective potential is exactly given by (3.18).

There is one further well known higher derivative term [59,60, 61, 62, 63, 64, 65, 66, 67, 68,

$$
\delta S_{3}=\int d^{10} x \sqrt{-g^{(10)}}\left(\frac{\alpha^{\prime 3} \zeta(3)}{3 \cdot 2^{12} \kappa_{10}^{2}} e^{-2 \varphi}+\frac{b_{1}}{2 \pi \alpha^{\prime}}\right) J_{0}
$$

where

$$
J_{0}=t^{M_{1} N_{1} \ldots M_{4} N_{4}} t_{M_{1}^{\prime} N_{1}^{\prime} \ldots M_{4}^{\prime} N_{4}^{\prime}} R^{M_{1}^{\prime} N_{1}^{\prime}}{ }_{M_{1} N_{1}} \ldots R^{M_{4}^{\prime} N_{4}^{\prime}} M_{4} N_{4}+\frac{1}{4} E_{8} .
$$

The tensor $t$ is defined by $t^{M_{1} \ldots M_{8}} A_{M_{1} M_{2}} \ldots A_{M_{7} M_{8}}=24 \operatorname{tr} A^{4}-6\left(\operatorname{tr} A^{2}\right)^{2}$ for antisymmetric tensors $A$, i.e. it does not contain the $\epsilon$-term [71]. If we assume that the dilaton $\varphi$ does not depend on the internal coordinates, this term does not contribute to the two-dimensional potential, as the integral of $J_{0}$ over a Calabi-Yau manifold vanishes [62,63, 72,

Unlike in the three-dimensional case we can not exclude that other higher derivative corrections might contribute to the potential at the same order of $\alpha^{\prime}$ as the kinetic terms

8 This can be clearly seen e.g. from the formulas of appendix A in [34]. 
of the RR-fields. The reason for this is the different $\alpha^{\prime}$-order of the various fluxes, that we have seen above. 9 This implies, for example, that a term $\sim F_{4}^{2} R^{3}$ would be of order $\mathcal{O}\left(\alpha^{\prime 6}\right)$, while the contribution of the kinetic term for $F_{8}$ is already of order $\mathcal{O}\left(\alpha^{\prime 7}\right)$. Having said this caveat, however, we now proceed calculating the effective potential, taking into account only the terms appearing in (2.30), (3.11) and (3.16). They give at least the leading contributions for each single flux.

Introducing the background fluxes (3.13) into $S_{10}+\delta S_{1}+\delta S_{2}$ results in the following two-dimensional action 10

$$
S_{2}=S_{2}^{(\mathrm{nf})}-\int d^{2} x \sqrt{-g^{(2)}} V
$$

Here $S_{2}^{(\mathrm{nf})}$ is the action (3.5) with no fluxes, whereas the scalar potential $V$ is given by

$$
\begin{aligned}
V=\frac{1}{4 \kappa_{10}^{2}}\left[\int_{Y_{4}} d^{8} \xi \sqrt{g^{(8)}}(\mid\right. & \left.F_{0}\right|^{2}+\left|F_{2}+F_{0} B\right|^{2}+\left|F_{4}+F_{2} \wedge B+\frac{1}{2} F_{0} B^{2}\right|^{2} \\
+\mid F_{6}+F_{4} & \wedge B+\frac{1}{2} F_{2} \wedge B^{2}+\left.\frac{1}{3 !} F_{0} B^{3}\right|^{2} \\
+\mid F_{8}+F_{6} & \left.\wedge B+\frac{1}{2} F_{4} \wedge B^{2}+\frac{1}{3 !} F_{2} \wedge B^{3}+\left.\frac{1}{4 !} F_{0} B^{4}\right|^{2}\right) \\
-\int_{Y_{4}}\left(2 F_{0} F_{8}-\right. & \left.\left.2 F_{2} \wedge F_{6}+F_{4} \wedge F_{4}\right)\right]
\end{aligned}
$$

Deriving this expression we used (2.31), (3.14), and (3.18), and also skipped the superscript of $B^{(0)}$ to avoid cluttering. To proceed further we use the relation $\int_{Y_{4}} d^{8} \xi \sqrt{g^{(8)}}\left|\omega_{p}\right|^{2}=$ $\int_{Y_{4}} \omega_{p} \wedge \star \omega_{p}$, valid for an arbitrary p-form $\omega_{p}$. With the help of the formulas for the Hodgedual of even degree forms on Calabi-Yau fourfolds given in appendix I, the potential (3.22)

9 This is also the reason, why the warp-factor might modify the potential even to the order we are working at.

10 Certainly there are also corrections from higher derivative terms to the kinetic terms displayed in (3.5), which we do not calculate here. 
can be expressed as

$$
\begin{gathered}
V=\frac{1}{4 \kappa_{10}^{2}} \mathcal{V}^{-1}\left[F_{0}^{2} \mathcal{V}^{2}+\frac{1}{36}\left(\int_{Y_{4}}\left(F_{2}+F_{0} B\right) \wedge J^{3}\right)^{2}-\frac{1}{2} \mathcal{V} \int_{Y_{4}}\left(F_{2}+F_{0} B\right)^{2} \wedge J^{2}\right. \\
-\mathcal{V} \int_{Y_{4}} F_{2,2} \wedge F_{2,2}+\mathcal{V} \int_{Y_{4}}\left(F_{2,2}+F_{2} \wedge B+\frac{1}{2} F_{0} B^{2}\right)^{2} \\
+\frac{1}{4}\left(g^{-1 A \bar{B}} \int_{Y_{4}}\left(F_{4}+F_{2} \wedge B+\frac{1}{2} F_{0} B^{2}\right) \wedge J \wedge e_{A}\right. \\
\quad \times \int_{Y_{4}}\left(F_{4}+F_{2} \wedge B+\frac{1}{2} F_{0} B^{2}\right) \wedge J \wedge e_{B} \\
\left.-\left(\int_{Y_{4}}\left(F_{4}+F_{2} \wedge B+\frac{1}{2} F_{0} B^{2}\right) \wedge J^{2}\right)^{2}\right) \\
+\frac{1}{4} g^{-1 A \bar{B}} \int_{Y_{4}}\left(F_{6}+F_{4} \wedge B+\frac{1}{2} F_{2} \wedge B^{2}+\frac{1}{3 !} F_{0} B^{3}\right) \wedge e_{A} \\
\times \int_{Y_{4}}\left(F_{6}+F_{4} \wedge B+\frac{1}{2} F_{2} \wedge B^{2}+\frac{1}{3 !} F_{0} B^{3}\right) \wedge e_{B} \\
+\left(\int_{Y_{4}}\left(F_{8}+F_{6} \wedge B+\frac{1}{2} F_{4} \wedge B^{2}+\frac{1}{3 !} F_{2} \wedge B^{3}+\frac{1}{4 !} F_{0} B^{4}\right)\right)^{2} \\
\left.-2 \mathcal{V} \int_{Y_{4}} F_{0} F_{8}+2 \mathcal{V} \int_{Y_{4}} F_{2} \wedge F_{6}\right] \\
-\frac{1}{\kappa_{10}^{2}} \int_{Y_{4}} F_{3,1} \wedge F_{1,3} .
\end{gathered}
$$

The last term in (3.23) is identical to the potential for the complex structure moduli appearing in three dimensions [37. As in that case it can be further rewritten by using [73]

$$
D_{\alpha} \Omega=\partial_{\alpha} \Omega+\left(\partial_{\alpha} K_{3,1}\right) \Omega=\Phi_{\alpha},
$$

where $\Phi_{\alpha}$ is the basis of $H^{3,1}\left(Y_{4}\right)$ and $K_{3,1}$ is the Kähler potential for the $(3,1)$-moduli defined in (3.9). With the help of (3.24) and (3.6) one derives

$$
-\frac{1}{\kappa_{10}^{2}} \int_{Y_{4}} F_{3,1} \wedge F_{1,3}=e^{K_{3,1}} g^{-1 \alpha \bar{\beta}} D_{\alpha} W D_{\bar{\beta}} \bar{W},
$$

where $W$ is precisely the chiral superpotential of [3], given in (3.1), if we set $\kappa_{10}=2 \pi$.

The potential for the Kähler moduli is more involved than in the three dimensional case, due to the additional background fluxes and the complexification of the Kähler moduli. It is a bit tedious but straightforward to verify that the first term in (3.23) can be expressed as

$$
\frac{1}{4 \kappa_{10}^{2}} \mathcal{V}^{-1}[\ldots]=\frac{1}{16} e^{K_{1,1}} g^{-1 A \bar{B}} D_{A} \widetilde{W} D_{\bar{B}} \overline{\widetilde{W}}
$$


where $K_{1,1}$ is the Kähler potential for the Kähler moduli, defined in (3.9), $\widetilde{W}$ is the twisted chiral superpotential of (3.2) and the Kähler covariant derivative is defined as $D_{A} \widetilde{W}=\partial_{A} \widetilde{W}+\left(\partial_{A} K_{1,1}\right) \widetilde{W}$. The formulas necessary for the derivation are collected in appendix I.

Obviously, defining

$$
\widehat{W}=\frac{1}{4} \widetilde{W}
$$

the potential takes the form

$$
V=e^{K_{1,1}} g^{-1 A \bar{B}} D_{A} \widehat{W} D_{\bar{B}} \overline{\widehat{W}}+e^{K_{3,1}} g^{-1 \alpha \bar{\beta}} D_{\alpha} W D_{\bar{\beta}} \bar{W}
$$

i.e. it does not contain any terms $\sim|W|^{2}$ or $\sim|\widehat{W}|^{2}$. The same phenomenon occurred in the three dimensional case for the potential of the complex structure moduli and it is closely related to the situation of four dimensional type IIB compactifications with 3 -form fluxes.

\section{3. (Non)-supersymmetric Vacua}

In the effective two-dimensional theory, the conditions for unbroken supersymmetry do not allow supersymmetric vacua with non-zero cosmological constant and require the following conditions to be satisfied [4:

$$
D_{A} \widetilde{W}=0, \quad D_{\alpha} W=0, \quad \widetilde{W}=0, \quad W=0 .
$$

This is similar to the situation discovered in three dimensions [3, 37]. Also as in the three-dimensional case, a 4 -form flux $F_{4} \sim \bar{\Omega}$ breaks supersymmetry without introducing a vacuum energy [25,2]. This is due to the fact that $W \neq 0$ but $V=0$ according to (3.25). A further flux with this property, $F_{4} \sim J^{2}$, has been found in [42]. It has a generalization in the two-dimensional situation at hand. Using (3.23) and the formulas from appendix I one shows that for $B=0$ an arbitrary combination of the following fluxes leads to a vanishing potential:

$$
F_{8}=\frac{F_{0}}{4 !} J^{4}, \quad F_{6}=-\frac{1}{3 !} F_{2} \wedge J^{2} \quad \text { with } \quad F_{2} \sim J, \quad F_{4} \sim J^{2} .
$$

Generically these fluxes break supersymmetry, because of $\widetilde{W} \neq 0$. However, the combination

$$
F_{8}=\frac{F_{0}}{4 !} J^{4} \quad \text { and } \quad F_{4}=\frac{F_{0}}{3 !} J^{2}
$$


leads to a vanishing $\widetilde{W}$ for $B=0$ and therefore to unbroken supersymmetry.

This shows a general feature of the fourfold models in type IIA string theory: they admit a rich vacuum structure due to the many possible Ramond-Ramond fluxes that one can introduce in the background. In fact, the non-trivial dependence of the potential on the geometric moduli of the Calabi-Yau fourfold generically allows one to stabilize all of them, including the volume modulus. This is in contrast to similar compactifications of M-theory and F-theory [3, 37,25], where the volume remains a flat direction of the tree-level potential.

To see that this problem does not occur in the type IIA theory, let us consider the simple case of a Calabi-Yau fourfold with $h^{1,1}\left(Y_{4}\right)=1.11$ Thus there is a single Kähler modulus $t=M+i a$. Furthermore let us assume that the (1,1)-form $e$ is normalized in such a way that the intesection number $\int e \wedge e \wedge e \wedge e=1$, so that $\mathcal{V}=\frac{1}{4 !} M^{4}$. Finally let us consider only nonzero 0 -form and 8 -form flux. In this case the potential (3.23) becomes

$$
V=\frac{1}{4 \kappa_{10}^{2}} M^{-4}\left(\frac{F_{0}^{2}}{4 !}\left(M^{2}+a^{2}\right)^{4}+2 F_{0}\left(\int_{Y_{4}} F_{8}\right)\left(a^{4}-M^{4}\right)+4 !\left(\int_{Y_{4}} F_{8}\right)^{2}\right) .
$$

The structure of the vacuum of (3.32) depends on the relative sign of the fluxes $F_{0}$ and $\int F_{8}$. If both have the same sign, then the only minimum of the potential $V$ is at:

$$
a=0, \quad M=\left(\frac{4 !}{F_{0}} \int_{Y_{4}} F_{8}\right)^{1 / 4}, \quad \mathcal{V}=\frac{1}{F_{0}} \int_{Y_{4}} F_{8} .
$$

In this minimum $V=0$, but supersymmetry is broken because of a non-vanishing $\widetilde{W}$, as was already discussed above. However, if one also turns on 4-form flux, the values (3.33) still lead to a minimum of the potential at $V=0$. In this case it is possible to find a supersymmetric minimum, if (3.31) and the remaining conditions of (3.29) are satisfied.

On the other hand, if $F_{0}$ and $\int F_{8}$ have opposite signs, the minimum of (3.32) occurs at:

$$
a=0, \quad M=\left(-\frac{4 !}{F_{0}} \int_{Y_{4}} F_{8}\right)^{1 / 4}, \quad \mathcal{V}=-\frac{1}{F_{0}} \int_{Y_{4}} F_{8} .
$$

Substituting this back into (3.32), one finds the value of the potential at the minimum:

$$
V_{\min }=\frac{1}{\kappa_{10}^{2}}\left|F_{0} \int_{Y_{4}} F_{8}\right|
$$

In particular, note that $V_{\min }>0$, and the minimum is classically stable.

11 Examples of such manifolds can be found e.g. in [74, 75]. 
This simple example should generalize to generic Calabi-Yau fourfolds and fluxes. Therefore, one can easily construct not only many supersymmetric vacua with fixed geometric moduli, but also configurations with a positive value of the scalar potential in the minimum. Such configurations obviously can not be supersymmetric, but perhaps can be useful in the quest for de Sitter vacua in string theory $776,77,78,79,80,81,82,83,1,12$ Unfortunately, Ramond-Ramond fluxes alone do not yield a $\mathrm{dS}_{2}$ vacuum. This is because the effective two-dimensional action one obtains from a four-fold compactification is in the string frame rather than in the Einstein frame and one can not generate a potential for the dilaton by turning on Ramond-Ramond fluxes only. One might hope to stabilize the dilaton by also introducing background NS-NS 3-form fluxes as in [25].13 It would be interesting to pursue this further.

\section{Examples}

Rather than demonstrating (3.26) in the general case, it is more instructive to consider a few specific examples, which illustrate the basic idea, and also help us to gain some intuition about the physical effects of different background fluxes.

\subsection{Reduction with 8-form Flux}

The simplest non-trivial example is when $F_{8}$ is the only non-trivial flux in the background. Then, from the dual Type IIA action (2.30), we expect the following scalar potential:

$$
V=\frac{1}{4 \kappa_{10}^{2}} \int_{Y_{4}} d \xi^{8} \sqrt{g^{(8)}}\left|F_{8}\right|^{2}=\frac{1}{4 \kappa_{10}^{2}} \mathcal{V}^{-1}\left(\int_{Y_{4}} F_{8}\right)^{2} .
$$

The Chern-Simons terms are not important in this case; they only give a tadpole cancellation condition:

$$
\chi\left(Y_{4}\right)=0
$$

Let us now demonstrate that this scalar potential is consistent with the proposed expression for the superpotential. Specifically, from (3.1) and (3.2) we get:

$$
W=0, \quad \widetilde{W}=\frac{1}{2 \pi} \int_{Y_{4}} F_{8} \in \mathbb{Z}
$$

12 We thank Andy Strominger for stimulating discussions on these aspects.

13 However, this would require to start with the massless type IIA theory, that depends on the NS-NS 2-form only via its field strength [30]. 
The integer constant $\widetilde{W}$ is nothing but the overall D0-brane charge "at infinity". Specifically, a D0-brane looks like a supersymmetric kink, or a BPS domain wall in the effective $\mathcal{N}=(2,2)$ two-dimensional theory. Moreover, it is magnetically charged with respect to the $F_{8}$, so as we go across this domain wall, the background value of the $F_{8}$-flux jumps by one unit. Hence, we can think of the $F_{8}$ (in fact, of any Ramond-Ramond flux) as being induced by D-brane charge placed at a large distance.

Motivated by the form of (3.26), let us evaluate:

$$
\begin{aligned}
g^{-1 A \bar{B}} D_{A} \widetilde{W} D_{\bar{B}} \overline{\widetilde{W}} & =g^{-1 A \bar{B}}\left(\partial_{A} K_{1,1}\right)\left(\partial_{\bar{B}} K_{1,1}\right)|\widetilde{W}|^{2} \\
& =4 g^{-1 A \bar{B}} \mathcal{V}^{-2} \mathcal{V}_{A} \mathcal{V}_{B}|\widetilde{W}|^{2} \\
& =4 \mathcal{V}^{-1} M^{A} \mathcal{V}_{A}|\widetilde{W}|^{2}=4|\widetilde{W}|^{2}
\end{aligned}
$$

Here we used some formulas from appendix I. This result is in complete agreement with (4.1) (if we use $\kappa_{10}=2 \pi$ ):

$$
V=\frac{1}{16} e^{K_{1,1}} g^{-1 A \bar{B}} D_{A} \widetilde{W} D_{\bar{B}} \overline{\widetilde{W}}=\frac{1}{4} \mathcal{V}^{-1}|\widetilde{W}|^{2}
$$

Notice, due to an inverse volume factor $\mathcal{V}^{-1}$, the scalar potential $V$ induced by the 8 -form flux has a minimum at large volume

$$
\mathcal{V} \rightarrow \infty
$$

\subsection{Reduction with 0-form Flux}

Another extreme example is when 0-form flux is the only non-vanishing R-R field in the background. Since $F_{0}$ plays the role of the mass parameter in type IIA supergravity, this case corresponds to compactification of massive type IIA supergravity on a Calabi-Yau fourfold $Y_{4}$.

Even though this case looks simple, in fact, it is the most difficult one. The reason is that when $F_{0}$ flux is non-zero, one has to take into account terms with all the $G_{2 n}^{(0)}$ fields in the dual Type IIA action (2.30), in order to reproduce the right dependence of the potential on the moduli fields. Specifically, $G_{2 n}^{(0)}$ depends on $F_{0}$ via

$$
G_{2 n}^{(0)}=\frac{1}{n !} F_{0} B^{n}+\ldots
$$

The superpotential (3.2) in this case reduces to

$$
\widetilde{W}=\frac{F_{0}}{2 \pi} \frac{1}{4 !} \int_{Y_{4}} \mathcal{K}^{4}=\frac{F_{0}}{2 \pi} \frac{1}{4 !} d_{A B C D} t^{A} t^{B} t^{C} t^{D}=\frac{F_{0}}{2 \pi} \mu,
$$


where we have introduced a holomorphic analog $\mu$ of the volume $\mathcal{V}$. Using the formulas from appendix I, it is easy to evaluate the right hand side of (3.26),

$$
\begin{aligned}
g^{-1 A \bar{B}} D_{A} \widetilde{W} D_{\bar{B}} \overline{\widetilde{W}} & =g^{-1 A \bar{B}}\left(\partial_{A} \widetilde{W}+\left(\partial_{A} K_{1,1}\right) \widetilde{W}\right)\left(\bar{\partial}_{B} \overline{\widetilde{W}}+\left(\bar{\partial}_{B} K_{1,1}\right) \overline{\widetilde{W}}\right) \\
& =g^{-1 A \bar{B}}\left(4 \frac{F_{0}}{2 \pi} \mu_{A}-\frac{2}{\mathcal{V}} \mathcal{V}_{A} \mu \frac{F_{0}}{2 \pi}\right)\left(4 \frac{F_{0}}{2 \pi} \bar{\mu}_{B}-\frac{2}{\mathcal{V}} \mathcal{V}_{B} \bar{\mu} \frac{F_{0}}{2 \pi}\right) \\
& =4\left(\frac{F_{0}}{2 \pi}\right)^{2} g^{-1 A \bar{B}}\left[4 \mu_{A} \bar{\mu}_{B}+\frac{1}{\mathcal{V}^{2}} \mathcal{V}_{A} \mathcal{V}_{B}|\mu|^{2}-2 \mu_{A} \mathcal{V}_{B} \frac{\bar{\mu}}{\mathcal{V}}-2 \mathcal{V}_{A} \bar{\mu}_{B} \frac{\mu}{\mathcal{V}}\right] \\
& =4\left(\frac{F_{0}}{2 \pi}\right)^{2}\left[4 g^{-1 A \bar{B}} \mu_{A} \bar{\mu}_{B}+|\mu|^{2}-2 M^{A} \mu_{A} \bar{\mu}-2 M^{B} \bar{\mu}_{B} \mu\right]
\end{aligned}
$$

For simplicity let us make the further assumption that $B=0$ (i.e. all $a^{A}=0$ ). Thus we have $\mu=\bar{\mu}=\mathcal{V}$ and $\mu_{A}=\bar{\mu}_{A}=\mathcal{V}_{A}$ and (4.6) simplifies such that

$$
V=\frac{1}{16} e^{K_{1,1}} g^{-1 A \bar{B}} D_{A} \widetilde{W} D_{\bar{B}} \overline{\widetilde{W}}=\frac{1}{4}\left(\frac{F_{0}}{2 \pi}\right)^{2} \mathcal{V}=\frac{1}{4} \mathcal{V}^{-1}|\widetilde{W}|^{2}
$$

This result agrees with (3.23) for the case under consideration and is very similar to (4.4) in the last subsection. Note, however, that now the scalar potential has a minimum at small volume:

$$
\mathcal{V} \rightarrow 0
$$

The moral is that $p$-form fluxes with small values of $p$ tend to minimize the volume of the compactification space, whereas $p$-form fluxes of high degree make the size of the compactification manifold grow. This is a very general phenomenon, known as attractor mechanism in higher-dimensional supergravity solutions [84,85,86]. Here, we can also arrange a very similar "attractor" behaviour, balancing effects of various fluxes and thus fixing the volume modulus as discussed at the end of the last section. Explicit examples of supersymmetric vacua were obtained in this way also in [4]. Fixing the volume seems to require $p$-form fluxes with different values of $p$, whose contributions to the (super)potential scale differently, compare e.g. the $J$-dependence of the various contributions to the superpotential $\widetilde{W}(3.2)$.

\subsection{Reduction with 4-form Flux}

In this subsection we would like to investigate the relation of the two-dimensional potential for non-vanishing 4-form flux to the corresponding three-dimensional potential. 
It has been found in [37], that the potential for M-theory on a fourfold is given by 14

$$
-\frac{1}{2 \pi} \int d^{3} x \sqrt{-g_{(M)}}\left[e^{K_{3,1}} g^{-1 \alpha \bar{\beta}} D_{\alpha} W D_{\bar{\beta}} \bar{W}+\mathcal{V}_{(\mathrm{M})}^{-1}\left(\frac{1}{4} g^{-1 A B} \partial_{A} \widetilde{W}_{(\mathrm{r})} \partial_{B} \widetilde{W}_{(\mathrm{r})}-\widetilde{W}_{(\mathrm{r})}^{2}\right)\right]
$$

where $\widetilde{W}_{(\mathrm{r})}$ is a real version of $\widetilde{W}$. To be more precise

$$
\widetilde{W}_{(\mathrm{r})}=\frac{1}{4} \int_{Y_{4}} \frac{F_{4}}{2 \pi} \wedge J_{(\mathrm{M})} \wedge J_{(\mathrm{M})}
$$

and the index at $g_{(M)}, \mathcal{V}_{(\mathrm{M})}$ and $J_{(\mathrm{M})}$ should remind at the fact, that the quantities are defined using the M-theory metric 15 , which is related to the type IIA string frame metric $g_{m n}^{(10)}$ via 87

$$
d s_{(11)}^{2}=g_{M N}^{(11)} d x^{M} d x^{N}=e^{-\frac{2 \varphi}{3}} g_{m n}^{(10)} d x^{m} d x^{n}+e^{\frac{4 \varphi}{3}}\left(d x^{11}+C_{m} d x^{m}\right)^{2} .
$$

Note that we have the same fluxes in M-theory and type IIA-theory, although in the Mtheory compactification it is the eleven dimensional analog of $d C_{3}$ from (2.1) which gets a flux and in the type IIA case it is the $d A_{3}$ introduced in (2.6). This is due to the fact that we are only considering 4-form flux, in which case the two different definitions of the flux indeed coincide.

Reducing (4.8) on the M-theory circle with radius $e^{\frac{2 \varphi}{3}}$ leads to the following contribution to the two-dimensional potential:

$$
-\int d^{2} x \sqrt{-g^{(2)}}\left[e^{K_{3,1}} g^{-1 \alpha \bar{\beta}} D_{\alpha} W D_{\bar{\beta}} \bar{W}+\left.e^{K_{1,1}}\left(g^{-1 A \bar{B}} D_{A} \widehat{W} D_{\bar{B}} \overline{\widehat{W}}\right)\right|_{\mathrm{B}=0}\right]
$$

where $\widehat{W}$ has been introduced in (3.27) and the second term only involves the real Kähler moduli. The contributions involving the moduli coming from the $B$-field, (c.f. (3.23)),

$$
-\int d^{2} x \sqrt{-g^{(2)}}\left[\frac{1}{16} e^{K_{1,1}}\left(g^{-1 A \bar{B}} \int_{Y_{4}} \frac{F_{4}}{2 \pi} \wedge B \wedge e_{A} \int_{Y_{4}} \frac{F_{4}}{2 \pi} \wedge B \wedge e_{B}+\left(\int_{Y_{4}} \frac{F_{4}}{2 \pi} \wedge B^{2}\right)^{2}\right)\right]
$$

14 This formula differs from the one given in [37, because we are interested here in the potential one gets without performing a Weyl-rescaling in $D=3$. It is this theory that gives the dilaton supergravity considered here upon reduction on a further circle. Furthermore in contrast to [37] we have chosen $\kappa_{11}=(2 \pi)^{3 / 2}$ here in order to get $\kappa_{10}=2 \pi$.

15 Implicitly also $g^{-1 A B}$ and $\partial_{A}$ depend on it. 
arise as follows. The three-dimensional action also contains the terms [37]

$$
-\frac{1}{2 \pi} \int d^{3} x \sqrt{-g_{(M)}}\left[\frac{1}{2(2 \pi)^{2}} \mathcal{V}_{(M)} g_{A B} F_{m n}^{A} F^{B m n}+\frac{1}{4 \pi} \epsilon^{m n p} \widetilde{W}_{A B} A_{m}^{A} F_{n p}^{B}\right]
$$

where $\widetilde{W}_{A B}=\frac{1}{4} \int_{Y_{4}} \frac{F_{4}}{2 \pi} \wedge e_{A} \wedge e_{B}$. We reduce this to two dimensions using the metric

$$
d s_{(3)}^{2}=g_{m n}^{(3)} d x^{m} d x^{n}=e^{-\frac{2 \varphi}{3}} g_{\mu \nu}^{(2)} d x^{\mu} d x^{\nu}+e^{\frac{4 \varphi}{3}}\left(d x^{3}+C_{\mu} d x^{\mu}\right)^{2},
$$

c.f. (4.10), which has the inverse

$$
g^{(3) m n}=\left(\begin{array}{cc}
e^{\frac{2 \varphi}{3}} g^{(2) \mu \nu} & -e^{\frac{2 \varphi}{3}} C^{\mu} \\
-e^{\frac{2 \varphi}{3}} C^{\nu} & e^{-\frac{4 \varphi}{3}}+e^{\frac{2 \varphi}{3}} C_{\rho} C^{\rho}
\end{array}\right) .
$$

Furthermore we take the Ansatz 88

$$
C_{m}^{A}=\left(A_{\mu}^{A}+C_{\mu} a^{A}, a^{A}\right)
$$

for the three-dimensional vectors. In this context the Ansatz for the external components can be interpreted as a change from the $C$-basis to the $A$-basis (2.8), because the $C_{m}^{A}$ arise from expanding the eleven-dimensional $C_{3}$ into the $(1,1)$-forms of $Y_{4}$, while $a^{A}$ are the expansion coefficients of $C_{3}$ into $e^{A} \wedge d x_{3}$, respectively of $B$ into $e^{A}$. As $C_{1}=A_{1}$ we see from (2.8) that the $A_{\mu}^{A}$ come from an expansion of $A_{3}$ into the (1,1)-forms of $Y_{4}$.

Introducing (4.14), (4.15) and (4.16) into (4.13) leads to the following terms in the two-dimensional effective action:

$$
\begin{aligned}
-\int d^{2} x \sqrt{-g^{(2)}} & {\left[\frac{1}{2(2 \pi)^{2}} \mathcal{V} g_{A \bar{B}}\left(F_{\mu \nu}^{A}+a^{A} F^{\mu \nu}\right)\left(F^{B \mu \nu}+a^{B} F^{\mu \nu}\right)\right.} \\
& \left.+\frac{1}{2 \pi} \epsilon^{\mu \nu} \widetilde{W}_{A B}\left(a^{A}\left(F_{\mu \nu}^{B}+a^{B} F_{\mu \nu}\right)-\frac{1}{2} a^{A} a^{B} F_{\mu \nu}\right)\right]
\end{aligned}
$$

where $F_{\mu \nu}$ is the field strength of $C_{\mu}$ and $F_{\mu \nu}^{A}$ that of $A_{\mu}^{A}$. In addition to (4.17) there is a term

$$
-\frac{1}{8(2 \pi)^{2}} \int d^{2} x \sqrt{-g^{(2)}} \mathcal{V} F_{\mu \nu} F^{\mu \nu}
$$

from the reduction of the three-dimensional Einstein-Hilbert action. Integrating out the vectorfields from (4.17) and (4.18), which do not have any dynamical degrees of freedom in two dimensions, leads exactly to the part of the potential involving the B-field moduli (4.12), if one uses the relation $B=a^{A} e_{A}$. Furthermore we notice that the equation of motion for $F_{\mu \nu}$ yields

$$
F_{\mu \nu}=4 \pi \epsilon_{\mu \nu} \mathcal{V}^{-1}\left(\int_{Y_{4}} \frac{F_{4}}{2 \pi} \wedge B^{2}\right)
$$


which amounts to a non-trivial fibration of the M-theory circle over the two-dimensional space-time.

\section{Acknowledgments}

We thank M. Berg, R. Kallosh, J. Louis, A. Strominger, and E. Witten for useful discussions. This research was partially conducted during the period S.G. served as a Clay Mathematics Institute Long-Term Prize Fellow. The work of S.G. is also supported in part by grant RFBR No. 01-02-17488, and the Russian President's grant No. 00-1599296. M.H. would like to thank the University of Princeton and especially I. Klebanov for hospitality at the beginning of the work. Moreover M.H. thanks the DFG for financial support and his work was supported in part by I.N.F.N., by the EC contract HPRN-CT2000-00122, by the EC contract HPRN-CT-2000-00148, by the INTAS contract 99-0-590 and by the MURST-COFIN contract 2001-025492. 


\section{Appendix I. Some Conventions and Useful Formulas}

In this appendix we summarize some conventions and useful relations used throughout the paper. We tried to make our conventions as close as possible to the existing notations in the literature, yet still consistent:

$\begin{array}{ll}Y_{4} & \text { Calabi-Yau fourfold, } \\ \Omega & \text { covariantly constant holomorphic 4-form on } Y_{4}, \\ e_{A} \in H^{2}\left(Y_{4}, \mathbb{R}\right) & \text { a basis of 2-forms on } Y_{4}, \\ J=M^{A} e_{A} & \text { Kähler form on } Y_{4}, \\ B^{(0)}=a^{A} e_{A} & \text { internal part of the NS-NS 2-form field, } \\ \mathcal{K}=J+i B^{(0)} & \text { complexified Kähler form on } Y_{4}, \\ t^{A}=M^{A}+i a^{A} & \text { complexified Kähler moduli of } Y_{4}, \\ Z^{\alpha} & \text { complex structure moduli of } Y_{4}, \\ H=d B & \text { NS-NS 3-form field strength, } \\ G_{2 n} & \text { Ramond-Ramond 2n-form field strength, } \\ \varphi & \text { ten-dimensional dilaton field, } \\ \mathcal{V} & \text { volume of } Y_{4}, \text { measured in the string frame metric. }\end{array}$

We also define

$$
\begin{aligned}
\mathcal{V} & =\frac{1}{4 !} \int_{Y_{4}} J \wedge J \wedge J \wedge J=\frac{1}{2^{4} 4 !} d_{A B C D}\left(t^{A}+\bar{t}^{A}\right)\left(t^{B}+\bar{t}^{B}\right)\left(t^{C}+\bar{t}^{C}\right)\left(t^{D}+\bar{t}^{D}\right) \\
\mathcal{V}_{A} & =\frac{1}{4 !} \int_{Y_{4}} e_{A} \wedge J \wedge J \wedge J=\frac{1}{4 !} d_{A B C D} M^{B} M^{C} M^{D}=\frac{1}{2} \partial_{A} \mathcal{V}=\frac{1}{2} \bar{\partial} \bar{A} \mathcal{V} \\
\mathcal{V}_{A B} & =\frac{1}{4 !} \int_{Y_{4}} e_{A} \wedge e_{B} \wedge J \wedge J=\frac{1}{4 !} d_{A B C D} M^{C} M^{D}=\frac{1}{3} \partial_{A} \partial_{B} \mathcal{V},
\end{aligned}
$$

where $d_{A B C D}$ are the classical intersection numbers (3.8). These quantities satisfy the following identities:

$$
\begin{aligned}
\mathcal{V}_{A} M^{A} & =\mathcal{V}, \\
\mathcal{V}_{A B} M^{B} & =\mathcal{V}_{A}, \\
g^{-1 A \bar{B}} \mathcal{V}_{B} & =\mathcal{V} M^{A}, \\
g^{-1 A \bar{B}} & =-\frac{1}{3} \mathcal{V} \mathcal{V}^{-1 A B}+\frac{4}{3} M^{A} M^{B},
\end{aligned}
$$

where the indices are raised with $\delta^{A B}$. 
Below we list some formulas for the Hodge duals of differential forms of even degree on $Y_{4}$ :

$$
\begin{aligned}
& \star \omega_{0,0}=\frac{1}{4 !} \omega_{0,0} J^{4}, \quad \star \omega_{4,4}=\mathcal{V}^{-1} \int_{Y_{4}} \omega_{4,4}, \\
& \star \omega_{1,1}=\frac{1}{36} \mathcal{V}^{-1}\left(\int_{Y_{4}} \omega_{1,1} \wedge J^{3}\right) J^{3}-\frac{1}{2} \omega_{1,1} \wedge J \wedge J, \\
& \star \omega_{3,3}=\frac{1}{4} \mathcal{V}^{-1} g^{-1 A \bar{B}}\left(\int_{Y_{4}} \omega_{3,3} \wedge e_{B}\right) e_{A}, \\
& \star \omega_{4,0}=\omega_{4,0}, \quad \star \omega_{3,1}=-\omega_{3,1}, \quad \star \omega_{1,3}=-\omega_{1,3}, \quad \star \omega_{0,4}=\omega_{0,4}, \\
& \star \omega_{2,2}=\omega_{2,2}+\frac{1}{4} \mathcal{V}^{-1}\left(g^{-1 A \bar{B}}\left(\int_{Y_{4}} \omega_{2,2} \wedge J \wedge e_{A}\right) J \wedge e_{B}-\left(\int_{Y_{4}} \omega_{2,2} \wedge J^{2}\right) J^{2}\right),
\end{aligned}
$$

which can be verified directly from the definition of the Hodge star on Calabi-Yau fourfolds

$$
\begin{aligned}
\star \omega_{p, q}= & \frac{1}{p ! q !(4-p) !(4-q) !} \omega_{i_{1} \ldots i_{p} \bar{\imath}_{1} \ldots \bar{\imath}_{q}} \epsilon^{i_{1} \ldots i_{p}}{\overline{j_{p+1}}}_{\bar{\jmath}_{p+1}} \epsilon^{\overline{1}_{1} \ldots \bar{\imath}_{q}}{ }_{j_{q+1} \ldots j_{D}} \\
& \times d \xi^{j_{q+1}} \wedge \ldots \wedge d \xi^{j_{D}} \wedge d \bar{\xi}^{\bar{j}_{p+1}} \wedge \ldots \wedge d \bar{\xi}^{\bar{j}_{D}} .
\end{aligned}
$$

Let us also introduce "holomorphic analogs" of $\mathcal{V}$ and $\mathcal{V}_{A}$ :

$$
\mu=\frac{1}{4 !} d_{A B C D} t^{A} t^{B} t^{C} t^{D}
$$

and

$$
\mu_{A}=\frac{1}{4 !} d_{A B C D} t^{B} t^{C} t^{D}, \quad \bar{\mu}_{A}=\frac{1}{4 !} d_{A B C D} \bar{t}^{B} \bar{t}^{C} \bar{t}^{D} .
$$




\section{Appendix II. Chiral Density Projectors In $\mathcal{N}=(2,2)$ Dilaton Supergravity}

In this appendix we find the chiral and twisted chiral density projectors in the $\mathcal{N}=$ $(2,2)$ dilaton supergravity constructed in [45]. As it was noticed in [45], unlike in gauged $\mathcal{N}=(2,2)$ supergravity [51], here the chiral projectors do not simply follow from the full superspace projector since $\widehat{\bar{\nabla}}^{2} \mathcal{L}$ is not a chiral superfield (for an arbitrary $\mathcal{L}$ ). Therefore, in order to solve the problem, we will have to study chiral superfields in the new $\mathcal{N}=(2,2)$ dilaton supergravity.

Let us first recall the supergravity algebra of the "old" $U(1)_{A} \otimes U(1)_{V}$ gauged dilaton supergravity [49]:

$$
\begin{aligned}
\left\{\nabla_{+}, \nabla_{+}\right\}=0 \quad, \quad\left\{\nabla_{-}, \nabla_{-}\right\}=0, \\
\left\{\nabla_{+}, \nabla_{\dot{+}}\right\}=i \nabla_{\ddagger} \quad, \quad\left\{\nabla_{-}, \nabla_{\dot{-}}\right\}=i \nabla_{=}, \\
\left\{\nabla_{+}, \nabla_{-}\right\}=-\frac{1}{2} \bar{R}\left(\mathcal{X}-i \mathcal{Y}^{\prime}\right) \quad, \quad\left\{\nabla_{+}, \nabla_{\dot{-}}\right\}=-\frac{1}{2} \bar{F}(\mathcal{X}-i \mathcal{Y}) .
\end{aligned}
$$

Here, we follow the notations of [45]. In particular, the Lorentz generators, $\mathcal{X}$, and Rsymmetry generators, $\mathcal{Y}$ and $\mathcal{Y}^{\prime}$, act on the covariant derivative $\nabla_{\alpha}$ as follows:

$$
\begin{aligned}
& {\left[\mathcal{X}, \nabla_{ \pm}\right]= \pm \frac{1}{2} \nabla_{ \pm} \quad, \quad\left[\mathcal{X}, \nabla_{ \pm}\right]= \pm \frac{1}{2} \nabla_{ \pm}} \\
& {\left[\mathcal{Y}, \nabla_{ \pm}\right]=-\frac{i}{2} \nabla_{ \pm} \quad, \quad\left[\mathcal{Y}, \nabla_{ \pm}\right]=+\frac{i}{2} \nabla_{ \pm}} \\
& {\left[\mathcal{Y}^{\prime}, \nabla_{ \pm}\right]=\mp \frac{i}{2} \nabla_{ \pm} \quad, \quad\left[\mathcal{Y}^{\prime}, \nabla_{ \pm}\right]= \pm \frac{i}{2} \nabla_{ \pm} .}
\end{aligned}
$$

De-gauging the $U(1)_{A} \otimes U(1)_{V}$ R-symmetry:

$$
\nabla_{\alpha} \rightarrow \hat{\nabla}_{\alpha}+\lambda_{\alpha} \mathcal{Y}+\tilde{\lambda}_{\alpha} \mathcal{Y}^{\prime}
$$

gives the "new" dilaton supergravity algebra:

$$
\begin{gathered}
\left\{\widehat{\nabla}_{+}, \widehat{\nabla}_{+}\right\}=i\left(\lambda_{+}+\widetilde{\lambda}_{+}\right) \widehat{\nabla}_{+}, \quad\left\{\widehat{\nabla}_{-}, \widehat{\nabla}_{-}\right\}=i\left(\lambda_{-}-\widetilde{\lambda}_{-}\right) \widehat{\nabla}_{-}, \\
\left\{\widehat{\nabla}_{+}, \widehat{\nabla}_{-}\right\}=-\frac{1}{2} \bar{R} \mathcal{X}+\frac{i}{2}\left(\lambda_{-}+\widetilde{\lambda}_{-}\right) \widehat{\nabla}_{+}+\frac{i}{2}\left(\lambda_{+}-\widetilde{\lambda}_{+}\right) \widehat{\nabla}_{-}, \\
\left\{\widehat{\nabla}_{+}, \widehat{\nabla}_{\dot{-}}\right\}=-\frac{1}{2} \bar{F} \mathcal{X}+\frac{i}{2}\left(\lambda_{\dot{-}}+\widetilde{\lambda}_{\dot{-}}\right) \widehat{\nabla}_{+}-\frac{i}{2}\left(\lambda_{+}-\widetilde{\lambda}_{+}\right) \widehat{\nabla}_{\dot{-}}, \\
\left\{\widehat{\nabla}_{+}, \widehat{\nabla}_{\dot{+}}\right\}=i \widehat{\nabla}_{\neq}+\frac{i}{2}\left(\lambda_{\dot{+}}+\widetilde{\lambda}_{\dot{+}}\right) \widehat{\nabla}_{+}-\frac{i}{2}\left(\lambda_{+}+\widetilde{\lambda}_{+}\right) \widehat{\nabla}_{\dot{+}},
\end{gathered}
$$




$$
\left\{\widehat{\nabla}_{-}, \widehat{\nabla}_{\dot{-}}\right\}=i \widehat{\nabla}_{=}+\frac{i}{2}\left(\lambda_{-}-\tilde{\lambda}_{\dot{-}}\right) \widehat{\nabla}_{-}-\frac{i}{2}\left(\lambda_{-}-\tilde{\lambda}_{-}\right) \widehat{\nabla}_{\dot{-}},
$$

where, in order to solve the constraints (Bianchi identities), one has:

$$
\begin{array}{cl}
\lambda_{+}=\widetilde{\lambda}_{+}=i\left(\widehat{\nabla}_{+} V\right) \quad, \quad \lambda_{-}=-\widetilde{\lambda}_{-}=i\left(\widehat{\nabla}_{-} V\right) \\
\lambda_{\dot{+}}=\widetilde{\lambda}_{\dot{+}}=-i\left(\widehat{\nabla}_{\dot{+}} V\right) \quad, \quad \lambda_{\dot{-}}=-\widetilde{\lambda}_{\dot{-}}=-i\left(\widehat{\nabla}_{\dot{-}} V\right)
\end{array}
$$

and

$$
\bar{R}=4 \widehat{\nabla}_{-} \widehat{\nabla}_{+} V \quad, \quad \bar{F}=4 \widehat{\nabla}_{-} \widehat{\nabla}_{+} V .
$$

In the following we will also need the definition of their lowest component fields [45]:

$$
F|=G, \quad R|=H
$$

In terms of the real unconstrained superfield $V$, we get the following supergravity algebra:

$$
\begin{gathered}
\left\{\widehat{\nabla}_{+}, \widehat{\nabla}_{+}\right\}=-2\left(\widehat{\nabla}_{+} V\right) \widehat{\nabla}_{+} \quad, \quad\left\{\widehat{\nabla}_{-}, \widehat{\nabla}_{-}\right\}=-2\left(\widehat{\nabla}_{-} V\right) \widehat{\nabla}_{-}, \\
\left\{\widehat{\nabla}_{+}, \widehat{\nabla}_{-}\right\}=-\frac{1}{2} \bar{R} \mathcal{X} \quad, \quad\left\{\widehat{\nabla}_{+}, \widehat{\nabla}_{-}\right\}=-\frac{1}{2} \bar{F} \mathcal{X}, \\
\left\{\widehat{\nabla}_{+}, \widehat{\nabla}_{\dot{+}}\right\}=i \widehat{\nabla}_{+}+\left(\widehat{\nabla}_{\dot{+}} V\right) \widehat{\nabla}_{+}+\left(\widehat{\nabla}_{+} V\right) \widehat{\nabla}_{\dot{+}} \\
\left\{\widehat{\nabla}_{-}, \widehat{\nabla}_{\dot{-}}\right\}=i \widehat{\nabla}_{=}+\left(\widehat{\nabla}_{\dot{-}} V\right) \widehat{\nabla}_{-}+\left(\widehat{\nabla}_{-} V\right) \widehat{\nabla}_{\dot{-}} .
\end{gathered}
$$

Let us consider the full superspace density projector:

$$
\begin{aligned}
& \int d^{2} x d^{4} \theta E^{-1} \mathcal{L}= \\
& =\int d^{2} x e^{-1}\left[\nabla^{2}+i \psi_{=}^{\dot{\perp}} \nabla_{+}-i \psi_{\ddagger}^{\dot{+}} \nabla_{-}+\left(-\frac{1}{2} \bar{H}-\psi_{\ddagger}^{\dot{-}} \psi_{=}^{\dot{+}}+\psi_{=}^{\dot{\Xi}} \psi_{\ddagger}^{\dot{+}}\right)\right] \bar{\nabla}_{\dot{+}} \bar{\nabla}_{\dot{-}} \mathcal{L} \mid= \\
& =\int d^{2} x e^{-1}\left[\left(\widehat{\nabla}_{+}-\left(\widehat{\nabla}_{+} V\right)\right)\left(\widehat{\nabla}_{-}-\left(\widehat{\nabla}_{-} V\right)\right)+i \psi_{=}^{\dot{\dot{ }}}\left(\widehat{\nabla}_{+}-\left(\widehat{\nabla}_{+} V\right)\right)-i \psi_{\neq}^{\dot{+}}\left(\widehat{\nabla}_{-}-\left(\widehat{\nabla}_{-} V\right)\right)+\right. \\
& \left.+\left(-\frac{1}{2} \bar{H}-\psi_{\ddagger}^{\dot{\dot{m}}} \psi_{=}^{\dot{\dot{ }}}+\psi_{\Xi}^{\dot{\dot{\Xi}}} \psi_{\ddagger}^{\dot{+}}\right)\right] \widehat{\bar{\nabla}}^{2} \mathcal{L} \mid= \\
& =\int d^{2} x e^{-1}\left[\widehat{\nabla}_{+} \widehat{\nabla}_{-}+i\left(\psi_{=}^{\dot{\perp}}-\lambda_{-}\right) \widehat{\nabla}_{+}-i\left(\psi_{\neq}^{\dot{+}}-\lambda_{+}\right) \widehat{\nabla}_{-}+\right. \\
& \left.+\left(-\frac{1}{4} \bar{H}-\psi_{\neq}^{\dot{-}} \psi_{=}^{\dot{+}}+\left(\psi_{=}^{\dot{-}}-\lambda_{-}\right)\left(\psi_{\ddagger}^{\dot{+}}-\lambda_{+}\right)\right)\right] \widehat{\nabla}_{\dot{+}} \widehat{\nabla}_{\dot{-}} \mathcal{L} \mid .
\end{aligned}
$$

Here, the first line is expressed in the gauged covariant derivative $\nabla_{\alpha}$, whereas the last few lines are expressed in terms of the covariant derivative $\widehat{\nabla}_{\alpha}$, which does not contain any gauge connection. 
In the "old" gauged supergravity the measures are related as:

$$
\int d^{2} x d^{4} \theta E^{-1} \mathcal{L}=\int d^{2} x d^{2} \theta \mathcal{E}^{-1} \bar{\nabla}^{2} \mathcal{L} \mid
$$

Furthermore, since for any superfield $\mathcal{L}$ we have

$$
\nabla_{\dot{+}}\left(\nabla_{\dot{+}} \nabla_{\dot{-}} \mathcal{L}\right)=\nabla_{\dot{-}}\left(\nabla_{\dot{+}} \nabla_{\dot{-}} \mathcal{L}\right)=0,
$$

we conclude that $\mathcal{L}_{c}=\bar{\nabla}^{2} \mathcal{L}$ is a chiral superfield, and we can interpret the expression in the first line of (II.9) as a chiral density projector:

$$
\begin{aligned}
& \int d^{2} x d^{4} \theta E^{-1} \mathcal{L}=\int d^{2} x e^{-1}\left[\nabla^{2}+i \psi_{=}^{\stackrel{\dot{\perp}}{=}} \nabla_{+}-i \psi_{\ddagger}^{\dot{+}} \nabla_{-}+\right. \\
& \left.+\left(-\frac{1}{2} \bar{H}-\psi_{\ddagger}^{\dot{\bar{y}}} \psi_{=}^{\dot{\dot{ \pm}}}+\psi_{=}^{\dot{\dot{=}}} \psi_{\ddagger}^{\dot{\dot{+}}}\right)\right] \nabla_{\dot{+}} \nabla_{\dot{-}} \mathcal{L}\left|=\int d^{2} x d^{2} \theta \mathcal{E}^{-1} \bar{\nabla}^{2} \mathcal{L}\right| .
\end{aligned}
$$

Hence, the chiral measure $\mathcal{E}^{-1}$ is given, essentially, by the expression in the square brackets:

$$
\begin{aligned}
& \int d^{2} x d^{2} \theta \mathcal{E}^{-1} \mathcal{L}_{c}=\int d^{2} x e^{-1}\left[\nabla^{2}+i \psi_{=}^{\dot{\check{ }}} \nabla_{+}-i \psi_{\mp}^{\dot{+}} \nabla_{-}+\right. \\
& \left.+\left(-\frac{1}{2} \bar{H}-\psi_{\ddagger}^{\dot{\dot{-}}} \psi_{=}^{\dot{+}}+\psi_{=}^{\dot{\dot{\Xi}}} \psi_{\ddagger}^{\dot{+}}\right)\right] \mathcal{L}_{c} \mid .
\end{aligned}
$$

This reasoning, however, can not be applied to the last lines in (II.9) in the new ungauged supergravity. The reason is that $\widehat{\nabla}^{2} \mathcal{L}$ is not a chiral superfield in this theory. This can be easily seen, for example, if one hits this expression with $\widehat{\nabla}_{\dot{+}}$ :

$$
\widehat{\nabla}_{\dot{+}}\left(\widehat{\nabla}_{\dot{+}} \widehat{\nabla}_{\dot{-}} \mathcal{L}\right)=-\left(\widehat{\nabla}_{\dot{+}} V\right) \widehat{\nabla}_{\dot{+}} \widehat{\nabla}_{\dot{-}} \mathcal{L} \neq 0 .
$$

This example suggests how to modify $\widehat{\bar{\nabla}}^{2} \mathcal{L}$ in order to make it a chiral superfield. Let us define:

$$
\mathcal{L}_{c}=e^{V} \widehat{\nabla}_{\dot{+}} \widehat{\nabla}_{\dot{-}} \mathcal{L}
$$

It is easy to check that $\mathcal{L}_{c}$ defined this way is, in fact, a chiral superfield in the un-gauged $\mathcal{N}=(2,2)$ supergravity theory:

$$
\begin{aligned}
& \widehat{\nabla}_{\dot{+}} \mathcal{L}_{c}=\widehat{\nabla}_{\dot{+}}\left(e^{V} \widehat{\nabla}_{\dot{+}} \widehat{\nabla}_{\dot{-}} \mathcal{L}\right)= \\
& =e^{V}\left(\widehat{\nabla}_{\dot{+}}+\left(\widehat{\nabla}_{\dot{+}} V\right)\right) \widehat{\nabla}_{\dot{+}} \widehat{\nabla}_{\dot{-}} \mathcal{L}= \\
& =e^{V}\left(\widehat{\nabla}_{\dot{+}}^{2}+\left(\widehat{\nabla}_{\dot{+}} V\right) \widehat{\nabla}_{\dot{+}}\right) \widehat{\nabla}_{\dot{-}} \mathcal{L}=\quad \text { (SUGRA algebra) } \\
& =e^{V}\left(-\left(\widehat{\nabla}_{\dot{+}} V\right) \widehat{\nabla}_{\dot{+}}+\left(\widehat{\nabla}_{\dot{+}} V\right) \widehat{\nabla}_{\dot{+}}\right) \widehat{\nabla}_{\dot{-}} \mathcal{L}=0 .
\end{aligned}
$$


In a similar way one can show:

$$
\begin{aligned}
& \widehat{\nabla}_{\dot{-}} \mathcal{L}_{c}=\widehat{\nabla}_{\dot{-}}\left(e^{V} \widehat{\nabla}_{\dot{+}} \widehat{\nabla}_{\dot{-}} \mathcal{L}\right)=\quad(\text { since } \mathcal{L} \text { is scalar }) \\
& =-\widehat{\nabla}_{\dot{-}}\left(e^{V} \widehat{\nabla}_{\dot{-}} \widehat{\nabla}_{\dot{+}} \mathcal{L}\right)=-e^{V}\left(\widehat{\nabla}_{\dot{-}}+\left(\widehat{\nabla}_{\dot{-}} V\right)\right) \widehat{\nabla}_{\dot{-}} \widehat{\nabla}_{\dot{+}} \mathcal{L}= \\
& =-e^{V}\left(\widehat{\nabla}_{\dot{-}}^{2}+\left(\widehat{\nabla}_{\dot{-}} V\right) \widehat{\nabla}_{\dot{-}}\right) \widehat{\nabla}_{\dot{+}} \mathcal{L}= \\
& =e^{V}\left(\left(\widehat{\nabla}_{\dot{-}} V\right) \widehat{\nabla}_{\dot{-}}-\left(\widehat{\nabla}_{\dot{-}} V\right) \widehat{\nabla}_{\dot{-}}\right) \widehat{\nabla}_{\dot{+}} \mathcal{L}=0 .
\end{aligned}
$$

By analogy with the definition (ㅍ.11) of the chiral density projector in the "old" gauged supergravity, in the new ungauged formalism we also want to find $\mathcal{E}^{-1}$, such that for $\mathcal{L}_{c}=\exp (V) \widehat{\bar{\nabla}}^{2} \mathcal{L}$ (with arbitrary superfield $\mathcal{L}$ ), the chiral superspace integral

$$
\int d^{2} x d^{2} \theta \mathcal{E}^{-1} \mathcal{L}_{c}=\int d^{2} x d^{2} \theta \mathcal{E}^{-1} e^{V} \widehat{\bar{\nabla}}^{2} \mathcal{L}
$$

could be written in terms of the full superspace integral. Specifically, we get:

$$
\begin{aligned}
& \int d^{2} x d^{4} \theta E^{-1} \mathcal{L}= \\
& =\int d^{2} x e^{-1}\left[\left(\widehat{\nabla}_{+}-\left(\widehat{\nabla}_{+} V\right)\right)\left(\widehat{\nabla}_{-}-\left(\widehat{\nabla}_{-} V\right)\right)+i \psi_{=}^{\dot{\dot{ }}}\left(\widehat{\nabla}_{+}-\left(\widehat{\nabla}_{+} V\right)\right)-i \psi_{\neq}^{\dot{\dot{q}}}\left(\widehat{\nabla}_{-}-\left(\widehat{\nabla}_{-} V\right)\right)+\right. \\
& \left.+\left(-\frac{1}{2} \bar{H}-\psi_{\ddagger}^{\dot{-}} \psi_{=}^{\dot{+}}+\psi_{=}^{\dot{\dot{\Xi}}} \psi_{\neq}^{\dot{+}}\right)\right] \widehat{\bar{\nabla}}^{2} \mathcal{L} \mid= \\
& =\int d^{2} x e^{-1}\left[\left(\widehat{\nabla}_{+}-\left(\widehat{\nabla}_{+} V\right)\right)\left(\widehat{\nabla}_{-}-\left(\widehat{\nabla}_{-} V\right)\right)+i \dot{\psi}_{=}^{\dot{\Xi}}\left(\widehat{\nabla}_{+}-\left(\widehat{\nabla}_{+} V\right)\right)-i \psi_{\neq}^{\dot{+}}\left(\widehat{\nabla}_{-}-\left(\widehat{\nabla}_{-} V\right)\right)+\right. \\
& \left.+\left(-\frac{1}{2} \bar{H}-\psi_{\neq}^{\dot{-}} \psi_{=}^{\dot{+}}+\psi_{=}^{\dot{\dot{ }}} \psi_{\neq}^{\dot{+}}\right)\right] \cdot e^{-V} \cdot e^{+V} \cdot \widehat{\nabla}_{\dot{+}} \widehat{\nabla}_{-} \mathcal{L} \mid= \\
& =\int d^{2} x e^{-1} \exp (-\varphi)\left[\left(\widehat{\nabla}_{+}-2\left(\widehat{\nabla}_{+} V\right)\right)\left(\widehat{\nabla}_{-}-2\left(\widehat{\nabla}_{-} V\right)\right)+i \dot{\doteq}\left(\widehat{\nabla}_{+}-2\left(\widehat{\nabla}_{+} V\right)\right)-\right. \\
& \left.-i \psi_{\neq}^{\dot{+}}\left(\widehat{\nabla}_{-}-2\left(\widehat{\nabla}_{-} V\right)\right)+\left(-\frac{1}{2} \bar{H}-\psi_{\ddagger}^{\dot{-}} \psi_{=}^{\dot{+}}+\psi_{=}^{\dot{\dot{ }}} \psi_{\ddagger}^{\dot{+}}\right)\right] \cdot e^{+V} \cdot \widehat{\nabla}_{\dot{+}} \widehat{\nabla}_{\dot{-}} \mathcal{L} \mid,
\end{aligned}
$$

where we pulled $e^{-V}$ all the way to the left. This resulted in the $\exp (-\varphi)$ factor and in the difference in the numerical coefficients.

Because $\mathcal{L}_{c}=e^{+V} \widehat{\nabla}_{\dot{+}} \widehat{\nabla}_{\dot{+}} \mathcal{L}$ is chiral in the new supergravity, we can write a general formula for any chiral $\mathcal{L}_{c}$ :

$$
\begin{aligned}
& \int d^{2} x d^{2} \theta \mathcal{E}^{-1} \mathcal{L}_{c}= \\
& =\int d^{2} x e^{-1} \exp (-\varphi)\left[\left(\widehat{\nabla}_{+}-2\left(\widehat{\nabla}_{+} V\right)\right)\left(\widehat{\nabla}_{-}-2\left(\widehat{\nabla}_{-} V\right)\right)+i \psi_{=}^{\dot{\perp}}\left(\widehat{\nabla}_{+}-2\left(\widehat{\nabla}_{+} V\right)\right)-\right. \\
& \left.-i \psi_{\ddagger}^{\dot{+}}\left(\widehat{\nabla}_{-}-2\left(\widehat{\nabla}_{-} V\right)\right)+\left(-\frac{1}{2} \bar{H}-\psi_{\ddagger}^{\dot{\bar{y}}} \psi_{=}^{\dot{+}}+\psi_{=}^{\dot{\dot{\Xi}}} \psi_{\ddagger}^{\dot{+}}\right)\right] \mathcal{L}_{c} \mid .
\end{aligned}
$$


Using the commutation relations (II.4) in the new supergravity algebra, we get:

$$
\begin{aligned}
& \int d^{2} x d^{2} \theta \mathcal{E}^{-1} \mathcal{L}_{c}= \\
& =\int d^{2} x e^{-1} \exp (-\varphi)\left[\widehat{\nabla}_{+} \widehat{\nabla}_{-}-2\left(\widehat{\nabla}_{+} V\right) \widehat{\nabla}_{-}+2\left(\widehat{\nabla}_{-} V\right) \widehat{\nabla}_{+}-\right.
\end{aligned}
$$

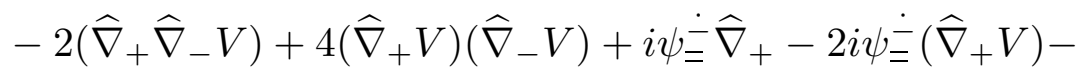

$$
\begin{aligned}
& \left.-i \psi_{\ddagger}^{\dot{+}} \widehat{\nabla}_{-}+2 i \psi_{\ddagger}^{\dot{\dot{+}}}\left(\widehat{\nabla}_{-} V\right)+\left(-\frac{1}{2} \bar{H}-\psi_{\neq}^{\dot{-}} \psi_{=}^{\dot{+}}+\psi_{=}^{\dot{-}} \psi_{\ddagger}^{\dot{+}}\right)\right] \mathcal{L}_{c} \mid= \\
& =\int d^{2} x e^{-1} \exp (-\varphi)\left[\widehat{\nabla}_{+} \widehat{\nabla}_{-}+2 i \lambda_{+} \widehat{\nabla}_{-}-2 i \lambda_{-} \widehat{\nabla}_{+}+\frac{\bar{R}}{2}-4 \lambda_{+} \lambda_{-}+\right. \\
& \left.+i \psi_{=}^{\dot{-}} \widehat{\nabla}_{+}-2 \psi_{=}^{\dot{\dot{\perp}}} \lambda_{+}-i \psi_{\ddagger}^{\dot{+}} \widehat{\nabla}_{-}+2 \psi_{\ddagger}^{\dot{+}} \lambda_{-}+\left(-\frac{1}{2} \bar{H}-\psi_{\ddagger}^{\dot{-}} \psi_{=}^{\dot{\dot{ }}}+\psi_{=}^{\dot{\dot{ }}} \psi_{\ddagger}^{\dot{\dot{+}}}\right)\right] \mathcal{L}_{c} \mid= \\
& =\int d^{2} x e^{-1} \exp (-\varphi)\left[\widehat{\nabla}_{+} \widehat{\nabla}_{-}+i\left(\psi_{=}^{\dot{\perp}}-2 \lambda_{-}\right) \widehat{\nabla}_{+}-i\left(\psi_{\ddagger}^{\dot{+}}-2 \lambda_{+}\right) \widehat{\nabla}_{-}-\right. \\
& \left.-\psi_{\ddagger}^{\dot{\dot{ }}} \psi_{=}^{\dot{\dot{+}}}+\left(\psi_{=}^{\dot{\dot{\Xi}}}-2 \lambda_{-}\right)\left(\psi_{\ddagger}^{\dot{\dot{+}}}-2 \lambda_{+}\right)\right] \mathcal{L}_{c} \mid \text {. }
\end{aligned}
$$

In the last equality we have used (II.7). Summarizing, we find the following chiral density projector formula:

$$
\begin{aligned}
& \int d^{2} x d^{2} \theta \mathcal{E}^{-1} \mathcal{L}_{c}=\int d^{2} x e^{-1} \exp (-\varphi)\left[\widehat{\nabla}_{+} \widehat{\nabla}_{-}+i\left(\psi_{=}^{\dot{-}}-2 \lambda_{-}\right) \widehat{\nabla}_{+}-\right. \\
& \left.-i\left(\psi_{\ddagger}^{\dot{+}}-2 \lambda_{+}\right) \widehat{\nabla}_{-}-\psi_{\ddagger}^{\dot{\dot{j}}} \psi_{=}^{\dot{+}}+\left(\psi_{=}^{\dot{\dot{ }}}-2 \lambda_{-}\right)\left(\psi_{\ddagger}^{\dot{+}}-2 \lambda_{+}\right)\right] \mathcal{L}_{c} \mid .
\end{aligned}
$$

Note, unlike the "old" chiral density projector, the right-hand side of this expression does not contain an $\bar{H}$-term. This means, however, that one runs into an obvious problem if one tries to incorporate the superpotential in the conventional way, i.e. via

$$
S=S_{0}+\int d^{2} x d^{2} \theta \mathcal{E}^{-1} W
$$

To see this let us evaluate the following superspace action for chiral superfields $\Phi_{i}$, c.f. (3.10) and [45]:

$$
S=\int d^{2} x d^{4} \theta E^{-1} \exp (-2 V) \exp (-K)+\int d^{2} x d^{2} \theta \mathcal{E}^{-1} W
$$

The terms relevant for the potential are

$$
L_{\mathrm{aux}} \sim e^{-2 \varphi-K}\left[\left|\frac{1}{2} H+i \bar{A}_{i} \frac{\partial K}{\partial \phi_{i}}\right|^{2}-\frac{\partial^{2} K}{\partial \phi_{i} \partial \bar{\phi}_{j}} A_{i} \bar{A}_{j}\right]-i \frac{\partial W}{\partial \phi_{i}} A_{i} e^{-\varphi}+\text { c.c. } .
$$

Integrating out the auxiliary field $H$, we get:

$$
L_{\mathrm{aux}} \sim e^{-2 \varphi-K}\left(\frac{\partial^{2} K}{\partial \phi_{i} \partial \bar{\phi}_{j}}\right) A_{i} \bar{A}_{j}-i\left(\frac{\partial W}{\partial \phi_{i}}\right) A_{i} e^{-\varphi}+\text { c.c. } .
$$


Finally, integrating out $A_{i}$ we obtain:

$$
L \sim e^{K} K_{\phi_{i} \bar{\phi}_{j}}^{-1}\left(\frac{\partial W}{\partial \phi_{i}}\right) \overline{\left(\frac{\partial W}{\partial \bar{\phi}_{j}}\right)},
$$

without the $|W|^{2}$-term and without any dependence on the dilaton. However, the derivatives that enter (II.21) are the ordinary partial derivatives, whereas we expect them to be replaced by suitable covariant derivatives in the theory of gravity. At the moment it is not clear to us, how to modify (III.20) in order to resolve this problem.

Similarly, one can obtain the twisted chiral density projector. We can define a twisted chiral field as (for arbitrary superfield $\mathcal{L}$ ):

$$
\mathcal{L}_{t c}=e^{V} \widehat{\nabla}_{\dot{+}} \widehat{\nabla}_{-} \mathcal{L} .
$$

Indeed, it satisfies:

$$
\widehat{\nabla}_{\dot{+}} \mathcal{L}_{t c}=\widehat{\nabla}_{-} \mathcal{L}_{t c}=0 .
$$

The proof is very similar to ([I.13) and (II.14):

$$
\begin{aligned}
& \widehat{\nabla}_{\dot{+}} \mathcal{L}_{t c}=\widehat{\nabla}_{\dot{+}}\left(e^{V} \widehat{\nabla}_{\dot{+}} \hat{\nabla}_{-} \mathcal{L}\right)= \\
& =e^{V}\left(\widehat{\nabla}_{\dot{+}}+\left(\widehat{\nabla}_{\dot{+}} V\right)\right) \widehat{\nabla}_{\dot{+}} \widehat{\nabla}_{-} \mathcal{L}= \\
& =e^{V}\left(\widehat{\nabla}_{\dot{+}}^{2}+\left(\widehat{\nabla}_{\dot{+}} V\right) \widehat{\nabla}_{\dot{+}}\right) \hat{\nabla}_{-} \mathcal{L}=0
\end{aligned}
$$

and

$$
\begin{aligned}
& \hat{\nabla}_{-} \mathcal{L}_{t c}=\hat{\nabla}_{-}\left(e^{V} \hat{\nabla}_{\dot{+}} \hat{\nabla}_{-} \mathcal{L}\right)= \\
& =-\hat{\nabla}_{-}\left(e^{V} \hat{\nabla}_{-} \hat{\nabla}_{\dot{+}} \mathcal{L}\right)=-e^{V}\left(\hat{\nabla}_{-}+\left(\widehat{\nabla}_{-} V\right)\right) \hat{\nabla}_{-} \widehat{\nabla}_{\dot{+}} \mathcal{L}= \\
& =-e^{V}\left(\widehat{\nabla}_{-}^{2}+\left(\hat{\nabla}_{-} V\right) \hat{\nabla}_{-}\right) \hat{\nabla}_{\dot{+}} \mathcal{L}=0 .
\end{aligned}
$$

The twisted chiral density projection formula in the $U(1)_{V}$ gauged supergravity theory has the following form, analogous to (III.9):

$$
\begin{aligned}
& \int d^{2} x d^{4} \theta E^{-1} \mathcal{L}= \\
& =\int d^{2} x e^{-1}\left[\nabla_{\dot{-}} \nabla_{+}-i \psi_{=}^{-} \nabla_{+}+i \psi_{\mp}^{\dot{+}} \nabla_{\dot{-}}+\left(\frac{1}{2} \bar{G}+\psi_{\mp}^{-} \psi_{=}^{\dot{+}}+\psi_{\mp}^{\dot{+}} \psi_{=}^{-}\right)\right] \nabla_{\dot{+}} \nabla_{-} \mathcal{L} \mid \\
& =\int d^{2} x e^{-1}\left[\left(\widehat{\nabla}_{\dot{-}}-\left(\widehat{\nabla}_{\dot{-}} V\right)\right)\left(\widehat{\nabla}_{+}-\left(\widehat{\nabla}_{+} V\right)\right)-i \psi_{=}^{-}\left(\widehat{\nabla}_{+}-\left(\widehat{\nabla}_{+} V\right)\right)+\right. \\
& \left.\quad+i \psi_{\mp}^{\dot{+}}\left(\widehat{\nabla}_{\dot{-}}-\left(\widehat{\nabla}_{\dot{-}} V\right)\right)+\left(\frac{1}{2} \bar{G}+\psi_{\mp}^{-} \psi_{=}^{\dot{+}}+\psi_{\mp}^{\dot{+}} \psi_{=}^{-}\right)\right] \widehat{\nabla}_{\dot{+}} \widehat{\nabla}_{-} \mathcal{L} \mid .
\end{aligned}
$$


Using the same arguments as above, we obtain the twisted chiral density projection formula in the dilaton $\mathcal{N}=(2,2)$ supergravity, c.f. ([I.15):

$$
\begin{aligned}
& \int d^{2} x d^{4} \theta E^{-1} \mathcal{L}= \\
& =\int d^{2} x e^{-1}\left[\left(\widehat{\nabla}_{\dot{-}}-\left(\widehat{\nabla}_{\dot{-}} V\right)\right)\left(\widehat{\nabla}_{+}-\left(\widehat{\nabla}_{+} V\right)\right)-i \psi_{=}^{-}\left(\widehat{\nabla}_{+}-\left(\widehat{\nabla}_{+} V\right)\right)+i \psi_{\neq}^{\dot{+}}\left(\widehat{\nabla}_{\dot{-}}-\left(\widehat{\nabla}_{\dot{-}} V\right)\right)+\right. \\
& \left.+\left(\frac{1}{2} \bar{G}+\psi_{\ddagger}^{-} \psi_{=}^{\dot{+}}+\psi_{\ddagger}^{\dot{+}} \psi_{=}^{-}\right)\right] \widehat{\nabla}_{\dot{+}} \widehat{\nabla}_{-} \mathcal{L} \mid= \\
& =\int d^{2} x e^{-1}\left[\left(\widehat{\nabla}_{\dot{-}}-\left(\widehat{\nabla}_{\dot{-}} V\right)\right)\left(\widehat{\nabla}_{+}-\left(\widehat{\nabla}_{+} V\right)\right)-i \psi_{=}^{-}\left(\widehat{\nabla}_{+}-\left(\widehat{\nabla}_{+} V\right)\right)+i \psi_{\neq}^{\dot{+}}\left(\widehat{\nabla}_{\dot{-}}-\left(\widehat{\nabla}_{\dot{-}} V\right)\right)+\right. \\
& \left.+\left(\frac{1}{2} \bar{G}+\psi_{\ddagger}^{-} \psi_{=}^{\dot{+}}+\psi_{\ddagger}^{\dot{+}} \psi_{=}^{-}\right)\right] \cdot e^{-V} \cdot e^{+V} \cdot \widehat{\nabla}_{\dot{+}} \widehat{\nabla}_{-} \mathcal{L} \mid= \\
& =\int d^{2} x e^{-1} \exp (-\varphi)\left[\left(\widehat{\nabla}_{\dot{-}}-2\left(\widehat{\nabla}_{\dot{-}} V\right)\right)\left(\widehat{\nabla}_{+}-2\left(\widehat{\nabla}_{+} V\right)\right)-i \psi_{=}^{-}\left(\widehat{\nabla}_{+}-2\left(\widehat{\nabla}_{+} V\right)\right)+\right. \\
& \left.+i \psi_{\ddagger}^{\dot{\dot{t}}}\left(\widehat{\nabla}_{\dot{-}}-2\left(\widehat{\nabla}_{\dot{-}} V\right)\right)+\left(\frac{1}{2} \bar{G}+\psi_{\ddagger}^{-} \psi_{=}^{\dot{\dot{+}}}+\psi_{\ddagger}^{\dot{+}} \psi_{=}^{-}\right)\right] \cdot e^{+V} \cdot \widehat{\nabla}_{\dot{+}} \widehat{\nabla}_{-} \mathcal{L} \mid .
\end{aligned}
$$

By replacing $e^{V} \widehat{\nabla}_{\dot{+}} \hat{\nabla}_{-} \mathcal{L}$ with an arbitrary twisted chiral superfield and following the same steps as in (II.16) and (II.17), we arrive at

$$
\begin{aligned}
& \int d^{2} x d^{2} \theta \widetilde{\mathcal{E}}^{-1} \mathcal{L}_{t c}=\int d^{2} x e^{-1} \exp (-\varphi)\left[\widehat{\nabla}_{\dot{-}} \widehat{\nabla}_{+}-i\left(\psi_{=}^{-}+2 \lambda_{\dot{-}}\right) \widehat{\nabla}_{+}+\right. \\
& \left.+i\left(\psi_{\mp}^{\dot{+}}-2 \lambda_{+}\right) \widehat{\nabla}_{\dot{-}}+\psi_{\ddagger}^{-} \psi_{=}^{\dot{+}}-\left(\psi_{=}^{-}+2 \lambda_{\dot{-}}\right)\left(\psi_{\mp}^{\dot{+}}-2 \lambda_{+}\right)\right] \mathcal{L}_{t c} \mid .
\end{aligned}
$$




\section{References}

[1] L.J. Dixon, "Some World Sheet Properties of Superstring Compactifications, on Orbifolds and Otherwise", Lectures given at the 1987 ICTP Summer Workshop in High Energy Phsyics and Cosmology, Trieste, Italy, Jun 29 - Aug 7, $198 \%$.

[2] C. Vafa, "Evidence for F-Theory", Nucl. Phys. B496 (1996) 403.

[3] S. Gukov, C. Vafa and E. Witten, "CFT's From Calabi-Yau Four-folds", Nucl. Phys. B584 (2000) 69, hep-th/9906070.

[4] S. Gukov, "Solitons, Superpotentials and Calibrations", Nucl. Phys. B574 (2000) 169, hep-th/9911011.

[5] A. Strominger, "Superstrings With Torsion", Nucl. Phys. B274 (1986) 253.

[6] R. Rohm and E. Witten, "The Antisymmetric Tensor Field in Superstring Theory", Ann. Phys. 170 (1986) 454.

[7] B. de Wit, D.J. Smit and N.D. Hari Dass, "Residual Supersymmetry of Compactified d = 10 Supergravity", Nucl. Phys. B283 (1987) 165.

[8] J. Polchinski and A. Strominger, "New Vacua for Type II String Theory", Phys. Lett. B388 (1996) 736, hep-th/9510227.

[9] E. Witten, "Strong Coupling Expansion of Calabi-Yau Compactification", Nucl. Phys. B471 (1996) 135, hep-th/9602070.

[10] J. Michelson, "Compactifications of Type IIB Strings to Four Dimensions with Nontrivial Classical Potential", Nucl. Phys. B495 (1997) 127, hep-th/9610151.

[11] A. Lukas, B. Ovrut and D. Waldram, "Stabilizing Dilaton and Moduli Vacua in String and M-Theory Cosmology," Nucl. Phys. B509 (1998) 169, hep-th/9611204.

[12] H.P. Nilles, M. Olechowski and M. Yamaguchi, "Supersymmetry Breaking and Soft Terms in M-Theory", Phys. Lett. B415 (1997) 24, hep-th/9707143.

[13] I. Antoniadis, E. Gava, K. S. Narain and T. R. Taylor, "Duality in Superstring Compactifications with Magnetic Field Backgrounds", Nucl. Phys. B511 (1998) 611, hepth/9708075.

[14] A. Lukas, B.A. Ovrut and D. Waldram, "On the Four-Dimensional Effective Action of Strongly Coupled Heterotic String Theory", Nucl. Phys. B532 (1998) 43, hepth/9710208.

[15] A. Lukas, B.A. Ovrut, K.S. Stelle and D. Waldram, "Heterotic M-Theory in Five Dimensions", Nucl. Phys. B552 (1999) 246, hep-th/9806051.

[16] T. R. Taylor and C. Vafa, "RR Flux on Calabi-Yau and Partial Supersymmetry Breaking", Phys. Lett. B474, 130 (2000), hep-th/9912152.

[17] K. Behrndt and S. Gukov, "Domain Walls and Superpotentials from M-Theory on Calabi-Yau Three-Folds", Nucl. Phys. B580 (2000) 225, hep-th/0001082.

[18] P. Mayr, "On Supersymmetry Breaking in String Theory and its Realization in Brane Worlds", Nucl. Phys. B593 (2001) 99, hep-th/0003198. 
[19] M. Spalinski and T.R. Taylor, "Branes and Fluxes in $D=5$ Calabi-Yau Compactifications of M Theory", Phys. Lett. B485 (2000) 263, hep-th/0004095.

[20] M. Grana and J. Polchinski, "Supersymmetric Three-Form Flux Perturbations on AdS(5)", Phys. Rev. D63 (2001) 026001, hep-th/0009211.

[21] S.S. Gubser, "Supersymmetry and F-Theory Realization of the Deformed Conifold with Three-Form Flux", hep-th/0010010.

[22] G. Dall'Agata, "Type IIB Supergravity Compactified on a Calabi-Yau Manifold with H-Fluxes", JHEP 0111:005 (2001), hep-th/0107264.

[23] G. Curio and A. Krause, "Four Flux and Warped Heterotic M Theory Compactifications", Nucl. Phys. B602 (2001) 172, hep-th/0012152.

[24] G. Curio, A. Klemm, D. Lüst and S. Theisen, "On the Vacuum Structure of Type II String Compactifications on Calabi-Yau Spaces with H-Fluxes", Nucl. Phys. B609 (2001) 3, hep-th/0012213.

[25] S.B. Giddings, S. Kachru and J. Polchinski, "Hierarchies from Fluxes in String Compactifications", hep-th/0105097.

[26] G. Curio, A. Klemm, B. Körs and D. Lüst, "Fluxes in Heterotic and Type II String Compactifications", Nucl. Phys. B620 (2002) 237, hep-th/0106155.

[27] G. Curio and A. Krause, "G-fluxes and Non-Perturbative Stabilisation of Heterotic M-Theory", hep-th/0108220.

[28] J. Louis and A. Micu, "Heterotic String Theory with Background Fluxes", Nucl. Phys. B626 (2002) 26, hep-th/0110187.

[29] G. Curio, B. Körs and D. Lüst, "Fluxes and Branes in Type II Vacua and M Theory Geometry with $G_{2}$ and $\operatorname{Spin}(7)$ Holonomy", hep-th/0111165.

[30] J. Louis and A. Micu, "Type II Theories Compactified on Calabi-Yau Threefolds in the Presence of Background Fluxes", hep-th/0202168.

[31] B. S. Acharya and B. Spence, "Flux, Supersymmetry and M Theory on 7-Manifolds," hep-th/0007213.

[32] B. Acharya, S. Gukov and X. de la Ossa, "Supersymmetry, G-flux and Spin(7) Manifolds", hep-th/0201227.

[33] C. Beasley and E. Witten, "A Note on Fluxes and Superpotentials in M-Theory Compactifications on Manifolds of $G_{2}$ Holonomy", hep-th/0203061.

[34] S. Kachru, M. Schulz and S. Trivedi, "Moduli Stabilization from Fluxes in a Simple IIB Orientifold," hep-th/0201028.

[35] E. Witten, "Non-Perturbative Superpotentials in String Theory," Nucl. Phys. B474 (1996) 343, hep-th/9604030.

[36] S. Gukov, Ph.D. Thesis, Princeton University, 2001.

[37] M. Haack and J. Louis, "M-theory Compactified on Calabi-Yau Fourfolds With Background Flux", Phys. Lett. B507 (2001) 296, hep-th/0103068. 
[38] K. Becker and M. Becker, "M-Theory on Eight-Manifolds", Nucl. Phys. B477 (1996) 155, hep-th/9605053.

[39] P. Mayr, "Mirror Symmetry, N = 1 Superpotentials and Tensionless Strings on CalabiYau Four-Folds", Nucl. Phys. B494 (1997) 489, hep-th/9610162.

[40] K. Dasgupta, G. Rajesh and S. Sethi, "M-Theory, Orientifolds and G-Flux", JHEP 9908:023 (1999), hep-th/9908088.

[41] B.R. Greene, K. Schalm and G. Shiu, "Warped Compactifications in M and F Theory", Nucl. Phys. B584 (2000) 480, hep-th/0004103.

[42] K. Becker and M. Becker, "Supersymmetry Breaking, M Theory and Fluxes", JHEP 0107:038 (2001), hep-th/0107044.

[43] W. Lerche, "Fayet-Iliopoulos Potentials from Fourfolds", JHEP 9711:004 (1997), hepth/9709146.

[44] P. Kaste, "On the Twisted Chiral Potential in $2 \mathrm{~d}$ and the Analogue of Rigid Special Geometry for 4-Folds", JHEP 9906:021 (1999), hep-th/9904218.

[45] S.J. Gates, Jr., S. Gukov and E. Witten, "Two Two-Dimensional Supergravity Theories from Calabi-Yau Four-Folds", Nucl. Phys. B584 (2000) 109, hep-th/0005120.

[46] M. Haack, J. Louis and M. Marquart, "Type IIA and Heterotic String Vacua in D=2", hep-th/0011075.

[47] N. Berkovits, S. Gukov and B.C. Vallilo, "Superstrings in 2D Backgrounds with R-R Flux and New Extremal Black Holes", Nucl. Phys. B614 (2001) 195, hep-th/0107140.

[48] E. Bergshoeff, R. Kallosh, T. Ortin, D. Roest and A. Van Proeyen, "New Formulations of D = 10 Supersymmetry and D8 - O8 Domain Walls", Class. Quant. Grav. 18 (2001) 3359, hep-th/0103233.

[49] S.J. Gates, Jr., M.T. Grisaru and M.E. Wehlau, "A Study of General 2D, N=2 Matter Coupled to Supergravity in Superspace, Nucl. Phys. B460 (1996) 579, hepth/9509021.

[50] M.T. Grisaru and M.E. Wehlau, "Prepotentials for $(2,2)$ Supergravity", Int. J. Mod. Phys. A10 (1995) 753, hep-th/9409043.

[51] M.T. Grisaru and M.E. Wehlau, "Superspace Measures, Invariant Actions, and Component Projection Formulae for (2,2) Supergravity", Nucl. Phys. B457 (1995) 219, hep-th/9508139.

[52] L.J. Romans, "Massive $N=2 A$ Supergravity in Ten Dimensions", Phys. Lett. B169 (1986) 374.

[53] S.J. Gates, Jr., C.M. Hull and M. Rocek, "Twisted Multiplets and New Supersymmetric Non-linear Sigma-Models", Nucl. Phys. B248 (1984) 157.

[54] M. Haack and J. Louis, "Duality in Heterotic Vacua With Four Supercharges", Nucl. Phys. B575 (2000) 107, hep-th/9912181.

[55] C. Vafa and E. Witten, "A One Loop Test of String Duality", Nucl. Phys. B447 (1995) 261, hep-th/9505053. 
[56] M.J. Duff, J.T. Liu and R. Minasian, "Eleven-Dimensional Origin of String-String Duality: A One Loop Test", Nucl. Phys. B452 (1995) 261, hep-th/9506126.

[57] S. Sethi, C. Vafa and E. Witten, "Constraints on Low-Dimensional String Compactification", Nucl. Phys. B480 (1996) 213, hep-th/9606122.

[58] K. Dasgupta and S. Mukhi, "A Note on Low-Dimensional String Compactifications", Phys. Lett. B398 (1997) 285, hep-th/9612188.

[59] M.B. Green and J.H. Schwarz, "Supersymmetrical Dual String Theory 2. Vertices and Trees", Nucl. Phys. B198 (1982) 252.

[60] M.T. Grisaru, A.E.M. van de Ven and D. Zanon, "Four-Loop $\beta$-Function for the $N=1$ and $N=2$ Supersymmetric Non-Linear Sigma Model in Two Dimensions", Phys. Lett. B173 (1986) 423; "Four Loop Divergences for the $N=1$ Supersymmetric Nonlinear Sigma Model in Two Dimensions", Nucl. Phys. B277 (1986) 409.

[61] M.T. Grisaru and D. Zanon, "Sigma Model Superstring Corrections to the EinsteinHilbert Action", Phys. Lett. B177 (1986) 347.

[62] D. Gross and E. Witten, "Superstring Modification of Einstein Equations", Nucl. Phys. B277 (1986) 1.

[63] M.D. Freeman, C.N. Pope, M.F. Sohnius and K.S. Stelle, "Higher Order Sigma-Model Counterterms and the Effective Action for Superstrings", Phys. Lett. B178 (1986) 199.

[64] Q. Park and D. Zanon, "More on Sigma Model Beta Functions and Low-Energy Effective Actions", Phys. Rev. D35 (1987) 4038.

[65] N. Sakai and Y. Tanii, "One-Loop Amplitudes and Effective Action in Superstring Theories", Nucl. Phys. B287 (1987) 457.

[66] I. Antoniadis, S. Ferrara, R. Minasian and K.S. Narain, " $R^{4}$ Couplings in $M$ and Type II Theories on Calabi-Yau Spaces", Nucl. Phys. B507 (1997) 571, hep-th/9707013.

[67] E. Kiritsis and B. Pioline, "On $R^{4}$ Threshold Corrections in Type IIB String Theory and (p,q) String Instantons", Nucl. Phys. B508 (1997) 509, hep-th/9707018.

[68] J.G. Russo and A.A. Tseytlin, "One-Loop Four Graviton Amplitude in Eleven Dimensional Supergravity", Nucl. Phys. B578 (2000) 139, hep-th/9707134.

[69] A.A. Tseytlin, " $R^{4}$ Terms in 11 Dimensions and Conformal Anomaly of $(2,0)$ Theory", Nucl. Phys. B584 (2000) 233, hep-th/0005072.

[70] M.B. Green and P. Vanhove, "D-Instantons, Strings and M-Theory", Phys. Lett. B408 (1997) 122, hep-th/9704145.

[71] J.H. Schwarz, "Superstring Theory", Phys. Rept. 89 (1982) 223.

[72] S. Frolov and A.A. Tseytlin, " $R^{4}$ Corrections to the Conifold and $G_{2}$-Holonomy Spaces", hep-th/0111128.

[73] A. Strominger, "Special Geometry", Comm. Math. Phys. 133 (1990) 163.

[74] http://hep.itp.tuwien.ac.at/ kreuzer/CY/

[75] http://www.th.physik.uni-bonn.de/th/Supplements/cy.html 
[76] C.M. Hull, "Timelike T-Duality, de Sitter Space, Large N Gauge Theories and Topological Field Theory", JHEP 9807:021 (1998), hep-th/9806146; "De Sitter Space in Supergravity and M Theory", JHEP 0111:012 (2001), hep-th/0109213.

[77] J. Maldacena and C. Nunez, "Supergravity Description of Field Theories on Curved Manifolds and a No Go Theorem", Int. J. Mod. Phys. A16 (2001) 822, hepth/0007018.

[78] A. Chamblin and N.D. Lambert, "De Sitter Space from M-Theory", Phys. Lett. B 508 (2001) 369, hep-th/0102159; "Zero-Branes, Quantum Mechanics and the Cosmological Constant", Phys. Rev. D 65 (2002) 066002, hep-th/0107031.

[79] V. Balasubramanian, P. Horava and D. Minic, "Deconstructing de Sitter", JHEP 0105:043 (2001), hep-th/0103171.

[80] E. Silverstein, "(A)dS Backgrounds from Asymmetric Orbifolds", hep-th/0106209.

[81] M. Spradlin, A. Strominger and A. Volovich, "Les Houches Lectures on De Sitter Space", hep-th/0110007.

[82] P. Berglund, T. Hübsch and D. Minic, "De Sitter Spacetimes from Warped Compactifications of IIB String Theory", hep-th/0112079.

[83] S. Kachru, J. Pearson and H. Verlinde, "Brane/Flux Annihilation and String Dual of a Nonsupersymmetric Field Theory", hep-th/0112197.

[84] S. Ferrara, R. Kallosh and A. Strominger, "N=2 Extremal Black Holes", Phys. Rev. D52 (1995) 5412, hep-th/9508072.

[85] S. Ferrara and R. Kallosh, "Supersymmetry and Attractors", Phys. Rev. D54 (1996) 1514, hep-th/9602136; "Universality of Supersymmetric Attractors", Phys. Rev. D54 (1996) 1525, hep-th/9603090.

[86] S. Ferrara, G.W. Gibbons and R. Kallosh, "Black Holes and Critical Points in Moduli Space", Nucl. Phys. B500 (1997) 75, hep-th/9702103.

[87] E. Witten, "String Theory Dynamics in Various Dimensions", Nucl. Phys. B443 (1995) 85, hep-th/9503124.

[88] S. Ferrara and S. Sabharwal, "Quaternionic Manifolds for Type II Superstring Vacua of Calabi-Yau Spaces", Nucl. Phys. B332 (1990) 317. 